Y. Kitaoka

Nagoya Math. J.

Vo. 74 (1979), 95-122

\title{
MODULAR FORMS OF DEGREE $n$ AND REPRESENTATION BY QUADRATIC FORMS
}

\author{
YOSHIYUKI KITAOKA
}

Let $A^{(m)}, B^{(n)}$ be positive definite integral matrices and suppose that $B$ is represented by $A$ over each $p$-adic integers ring $Z_{p}$. Using the circle method or theory of modular forms in case of $n=1, B$, if sufficiently large, is represented by $A$ provided that $m \geq 5$. The approach via the theory of modular forms has been extended by [7] to Siegel modular forms to obtain a partial result in the particular case when $n=2, m \geq 7$. On the other hand Kneser gave an arithmetic approach in case of $n=1$ in his lectures [4] . Using this idea we proved that $B$ is represented by $A$ over $Z$ provided that $m \geq 2 n+3$ and the minimum of $B$ is sufficiently large [2]. Our aim here is to give an analytic proof in the case when $A$ is an even unimodular positive definite matrix. Under the algebraic preparations of $\S 1$ we give the Fourier expansion of Eisenstein series in the sense of Klingen and estimate coefficients from above. In $\S 3$ we estimate Fourier coefficients of usual Eisenstein series from above and below and it is applied to our problem in $\S 4$.

Notations. Let $H_{n}$ be the space of $n \times n$ complex symmetric matrices $Z$ with positive real $Y$. Let $\Gamma_{n}$ denote the group of integral $2 n \times 2 n$ matrices $M$ satisfying

$$
M I^{t} M=I, \quad I=\left(\begin{array}{cc} 
& 1_{n} \\
-1_{n} &
\end{array}\right)
$$

For $M \in \Gamma_{n}$ we put $M=\left(\begin{array}{ll}A_{M} & B_{M} \\ C_{M} & D_{M}\end{array}\right)$ where $A_{M}, \cdots, D_{M}$ are $n \times n$ matrices. For $1 \leq r \leq n-1$ we define the subgroup $\Delta_{n, r}$ of $\Gamma_{n}$ as the group of all $M \in \Gamma_{n}$ whose elements in the first $n+r$ colums and last $n-r$ rows vanish. The transposed matrix of a matrix $M$ is denoted by ${ }^{t} M$. We don't use the usual convention $A[B]={ }^{t} B A B . \quad \sigma$ stands for the trace of matrices. $e(x)$ means $\exp (2 \pi i x)$.

Received June 7, 1978. 
$\$ 1$.

Through this section we fix natural numbers $r, n$ which satisfy $1 \leq r$ $\leq n-1$. For a matrix $M \in M_{p, q}(C)(p \geq n-r)$ we denote by $\tilde{M}$ the last $n-r$ rows of $M$, i.e., $M=\left(\begin{array}{c}* \\ \tilde{M}\end{array}\right)$. For $M \in M_{n}(C)$ we decompose $M$ as $M$ $=\left(\begin{array}{ll}M_{1} & M_{2} \\ M_{3} & M_{4}\end{array}\right)$ where $M_{1} \in M_{r}(C), M_{2} \in M_{r, n-r}(C), M_{3} \in M_{n-r, r}(C), M_{4} \in M_{n-r}(C)$. $M_{i}$ are used in this sense if we don't refer.

Lemma 1. For $M, N \in \Gamma_{n}, \Delta_{n, r} M=\Delta_{n, r} N$ is equivalent to $\tilde{M}=g \tilde{N}$ for some $g \in G L(n-r, Z)$.

Proof. Let $M, N$ be elements of $\Gamma_{n}$. Suppose that $M=K N$ where $K=\left(\begin{array}{cc|cc}* & * & \\ \hline * & * & * & * \\ 0 & 0 & 0 & D_{4}\end{array}\right) \in \Delta_{n, r}$. Then we have $\tilde{M}=D_{4} \tilde{N}$. Since $K$ is a unimodular matrix, $D_{4}$ is unimodular. Conversely, suppose that $\tilde{M}=g \tilde{N}$ for

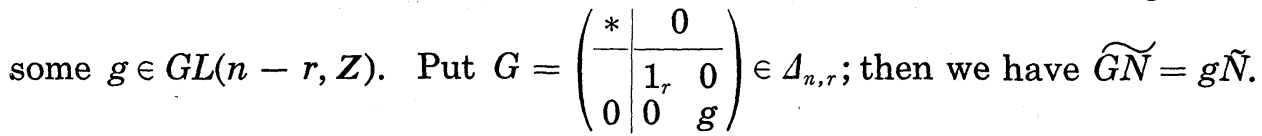
Hence we may assume $\tilde{M}=\tilde{N}$. Then $\widetilde{M N}^{-1}=\left(0^{(n+r, n-r)}, 1_{n-r}\right)$ holds. Hence $M N^{-1} \in \Delta_{n, r}$.

Q.E.D.

Lemma 2. For an element $N \in \Gamma_{n}$ with rank $\tilde{C}_{N}<n-r$, there is an element $M$ in $\Delta_{n, n-1}$ such that $\Delta_{n, r} N \ni M\left(\begin{array}{cc}U & 0 \\ 0 & { }^{t} U^{-1}\end{array}\right)$ for some $U \in G L(n, Z)$.

Proof. By the assumption rank $\tilde{C}_{N}<n-r$ there are unimodular matrices $g \in G L(n-r, Z), V \in G L(n, Z)$ such that the last row of $g \tilde{C}_{N} V$

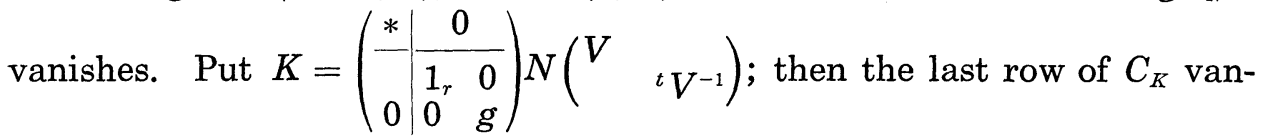
ishes and the elements of the last row of $D_{K}$ are relatively prime. Taking a unimodular matrix $W \in G L(n, Z)$ such that $D_{K} W=\left(\begin{array}{c}* \\ 0 \cdots 0\end{array}\right)$, we put $M=K\left(\begin{array}{ll}{ }^{t} W^{-1} & \\ & W\end{array}\right)$; then we have $M \in \Delta_{n, n-1}$ since the last row of $M$ is $(0, \cdots, 0,1)$. We may take $V^{t} W^{-1}$ as $U$.

Q.E.D.

Lemma 3. If ${ }^{t} A C$ is symmetric for $A, C \in M_{m}(Z)$, then there is a symmetric coprime pair $\left(\mathfrak{S}^{(m)}, \mathfrak{D}^{(m)}\right)$ such that $\mathfrak{\complement} A+\mathfrak{D} C=0$.

Proof. If $C=0$, then we may take $\mathfrak{C}=0, \mathfrak{D}=1_{m}$. Suppose $C \neq 0$. First we assume $|C| \neq 0$. Then $A C^{-1}$ is a symmetric rational matrix.

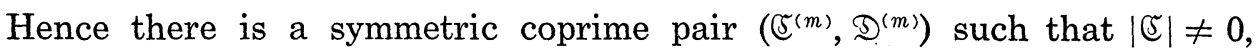


$\mathfrak{S}^{-1} \mathfrak{D}=-A C^{-1}$ (p. 166 in [5]). Thus we have $\mathfrak{S} A+\mathfrak{D} C=0 . \quad$ Next we assume $|C|=0$; then there are unimodular matrices $g_{1}, g_{2} \in G L(m, Z)$ such that $g_{1} C g_{2}^{-1}=c=\left(\begin{array}{cc}c_{1}^{(s)} & 0 \\ 0 & 0\end{array}\right)\left(\left|c_{1}\right| \neq 0, s \geq 1\right)$. Putting $a={ }^{t} g_{1}^{-1} A g_{2}^{-1}$, we have ${ }^{t} a c={ }^{t} g_{2}^{-1}{ }^{t} A C g_{2}^{-1}$ and this is a symmetric matrix. Decompose $a$ as $a=$ $\left(\begin{array}{ll}a_{1}^{(s)} & a_{2} \\ a_{3} & a_{4}\end{array}\right) . \quad{ }^{t} a c={ }^{t} c a$ implies that ${ }^{t} a_{1} c_{1}$ is symmetric and ${ }^{t} a_{2} c_{1}=0$ and so $a_{2}=0$. Take a symmetric coprime pair $\left(\mathfrak{S}_{1}^{(s)}, \mathfrak{D}_{1}^{(s)}\right)$ such that $\mathfrak{S}_{1} a_{1}+\mathfrak{D}_{1} c_{1}$ $=0$, and put $\mathfrak{S}=\left(\begin{array}{ll}\mathfrak{S}_{1} & \\ & 0\end{array}\right) \cdot \mathfrak{D}=\left(\begin{array}{ll}\mathfrak{D}_{1} & \\ & 1_{m-s}\end{array}\right)$. Then $(\mathfrak{S}, \mathfrak{D})$ is a symmetric coprime pair and

$$
\mathfrak{S} a+\mathfrak{D c}=\left(\begin{array}{ll}
\mathfrak{S}_{1} & 0 \\
0 & 0
\end{array}\right)\left(\begin{array}{ll}
a_{1} & 0 \\
a_{3} & a_{4}
\end{array}\right)+\left(\begin{array}{ll}
\mathfrak{D}_{1} & \\
& 1
\end{array}\right)\left(\begin{array}{ll}
c_{1} & \\
& 0
\end{array}\right)=0 .
$$

On the other hand, $\sqrt{ } a+\mathfrak{D} c=\left(\mathbb{\complement}^{t} g_{1}^{-1} A+\mathfrak{D} g_{1} C\right) g_{1}^{-1}$ implies $\mathfrak{C}^{t} g_{2}^{-1} A+\mathfrak{D} g_{1} C$ $=0$. Here it is easy to see that $\left(\complement^{t} g_{1}^{-1}, \mathfrak{D} g_{1}\right)$ is a symmetric coprime pair. This completes the proof.

Q.E.D.

Lemma 4. For $M \in \Gamma_{n}$ there are a unimodular matrix $U \in G L(n, Z)$ and $N \in \Delta_{n, r} M\left(\begin{array}{ll}{ }^{t} U & \\ & U^{-1}\end{array}\right)$ such that the first $r$ colums of $C_{N}$ vanishes.

Proof. Take $g \in G L(n-r, Z), U \in G L(n, Z)$ such that $g \tilde{C}_{M}{ }^{t} U=\left(0, C_{4}\right)$;

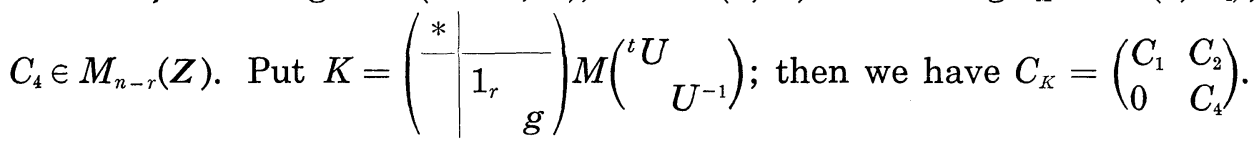
${ }^{t} A C={ }^{t} C A$ implies ${ }^{t} A_{1} C_{1}={ }^{t} C_{1} A_{1}$ where we put $A=A_{K}, C=C_{K}$. By Lemma 3 there is a symmetric coprime pair $\left(\mathfrak{\Im}_{1}^{(r)}, \mathfrak{D}_{1}^{(r)}\right)$ such that $\mathfrak{\Im}_{1} A_{1}+$ $\mathfrak{D}_{1} C_{1}=0$. Take an element $G \in \Delta_{n, r}$ such that $C_{G}=\left(\begin{array}{ll}\mathfrak{S}_{1} & 0 \\ 0 & 0\end{array}\right), D_{G}=\left(\begin{array}{ll}\mathfrak{D}_{1} & 0 \\ 0 & 1\end{array}\right)$; then $C_{G K}=\left(\begin{array}{ll}\mathfrak{S}_{1} & 0 \\ 0 & 0\end{array}\right)\left(\begin{array}{ll}A_{1} & A_{2} \\ A_{3} & A_{4}\end{array}\right)+\left(\begin{array}{ll}\mathfrak{D}_{1} & 0 \\ 0 & 1\end{array}\right)\left(\begin{array}{ll}C_{1} & C_{2} \\ & C_{4}\end{array}\right)=\left(\begin{array}{ll}0 & * \\ 0 & *\end{array}\right)$. This completes the proof.

Q.E.D.

We put $P_{n, r}=\left\{A=\left(\begin{array}{ll}A_{1}^{(r)} & A_{2} \\ A_{3} & A_{4}\end{array}\right) \in G L(n, Z) \mid A_{3}=0\right\}$.

Lemma 5. Let $M, N$ be elements of $\Gamma_{n}$ such that $\left(\begin{array}{l}C_{1} \\ C_{3}\end{array}\right)=\left(\begin{array}{l}C_{1}^{\prime} \\ C_{3}^{\prime}\end{array}\right)=0$, $\left|C_{4}\right|\left|C_{4}^{\prime}\right| \neq 0$ where we put $C_{M}=\left(\begin{array}{ll}C_{1} & C_{2} \\ C_{3} & C_{4}\end{array}\right), C_{N}=\left(\begin{array}{ll}C_{1}^{\prime} & C_{2}^{\prime} \\ C_{3}^{\prime} & C_{4}^{\prime}\end{array}\right)$. If $K M\left(\begin{array}{c}{ }^{t} V \\ V^{-1}\end{array}\right)=$ $N\left({ }^{t} U{ }^{-1}\right)$ for $K \in \Delta_{n, r}, U, V \in G L(n, Z)$, then we have $C_{K}=0,{ }^{t} U \in P_{n, r}{ }^{t} V$.

Proof. Put $W={ }^{t} U^{t} V^{-1}$; then $C_{K M}=C_{K} A_{M}+D_{K} C_{M}=C_{N} W$. Putting $A_{M}=\left(\begin{array}{ll}A_{1} & A_{2} \\ A_{3} & A_{4}\end{array}\right), C_{K}=\left(\begin{array}{cc}\mathfrak{S}_{1} & 0 \\ 0 & 0\end{array}\right), D_{K}=\left(\begin{array}{ll}\mathfrak{D}_{1} & \mathfrak{D}_{2} \\ & \mathfrak{D}_{4}\end{array}\right), W=\left(\begin{array}{ll}W_{1} & W_{2} \\ W_{3} & W_{4}\end{array}\right)$, we have 
$C_{4}^{\prime} W_{3}=0$ and $\mathfrak{\complement}_{1} A_{1}=C_{2}^{\prime} W_{3}$. The assumption $\left|C_{4}^{\prime}\right| \neq 0$ implies $W_{3}=0$. Hence ${ }^{t} U=W^{t} V \in P_{n, r}{ }^{t} V$ and $\mathcal{E}_{1} A_{1}=0$. We have only to prove $\left|A_{1}\right| \neq 0$. Since ${ }^{t} A_{M} C_{M}=\left(\begin{array}{cc}*{ }^{t} A_{1} C_{2}+{ }^{t} A_{3} C_{4} \\ 0 & *\end{array}\right)$ is symmetric, we have ${ }^{t} A_{1} C_{2}+{ }^{t} A_{3} C_{4}=0$ and $A_{3}=-{ }^{t} C_{4}^{-1}{ }^{t} C_{2} A_{1}$. Since the rank of the first $r$ colums of $M$ is $r$, we have $r=\operatorname{rank}\left(\begin{array}{l}A_{1} \\ A_{3}\end{array}\right)=\operatorname{rank}\left(\begin{array}{c}1_{r} \\ -{ }^{t} C_{4}^{-1 t} C_{2}\end{array}\right) A_{1} \leq \operatorname{rank} A_{1} \leq r$. Thus we have $\operatorname{rank} A_{1}=r$, i.e., $\left|A_{1}\right| \neq 0$ and so $\mathfrak{\complement}_{1}=0$.

Q.E.D.

The following lemma is a key in this paper.

Lemma 6. Let $M$ be an element of $\Gamma_{n}$ with $\operatorname{rank} \tilde{C}_{M}=n-r$. Then there is an element $N \in \Delta_{n, r} M$ such that $\left|C_{N}\right| \neq 0,\left(A_{N} C_{N}^{-1}\right)_{1} \equiv 0 \bmod 1$.

Proof. By virtue of Lemma 4 there exist $P \in \Gamma_{n}, U \in G L(n, Z)$ such that $\Delta_{n, r} M \ni P\left(\begin{array}{ll}{ }^{t} U & \\ & U^{-1}\end{array}\right), C_{P}=\left(\begin{array}{ll}0 & C_{2} \\ 0 & C_{4}\end{array}\right) . \quad \operatorname{rank} \tilde{C}_{M}=n-r$ yields $\left|C_{4}\right| \neq 0$. Put $A_{P}=\left(\begin{array}{ll}A_{1} & A_{2} \\ A_{3} & A_{4}\end{array}\right)$; then $\left|A_{1}\right| \neq 0$ holds as in the proof of Lemma 5 . Take $K=\left(\begin{array}{cc|c}1_{n} & 0 \\ \hline 1_{r} & 0 & 1_{n} \\ 0 & 0 & 1_{n}\end{array}\right) \in \Delta_{n, r}$ and put $K P=N^{\prime}$. Then $A_{N^{\prime}}=A_{P}, C_{N^{\prime}}=$ $\left(\begin{array}{ccc}A_{1} & A_{2}+C_{2} \\ 0 & C_{4}\end{array}\right)$ imply $\left|C_{N^{\prime}}\right| \neq 0,\left(A_{N^{\prime}} C_{N^{\prime}}^{-1}\right)_{1}=1_{r}$. Taking $N^{\prime}\left({ }^{t} U U^{-1}\right)$ as $N$, we have $\left|C_{N}\right| \neq 0,\left(A_{N} C_{N}^{-1}\right)_{1}=1_{r}$ and $\Delta_{n, r} M \ni N$.

Q.E.D.

Lemma 7. Let $C_{4}, D_{3}, D_{4}$ be elements of $M_{n-r}(Z), M_{n-r, r}(Z), M_{n-r}(Z)$ respectively. Suppose that $\left|C_{4}\right| \neq 0, C_{4}{ }^{t} D_{4}$ is symmetric and $\left(C_{4}, D_{3}, D_{4}\right)$ is primitive. Then there is a symmetric coprime pair $\left(C^{(n)}, D^{(n)}\right)$ such that $\tilde{C}$ $=\left(0, C_{4}\right), \tilde{D}=\left(D_{3} D_{4}\right)$.

Proof. Since rank $\left(C_{4}, D_{4}\right)=n-r$, there exist matrices $U_{4} \in M_{n-r}(Z)$, $V \in G L(2(n-r), Z)$ such that $\left(C_{4}, D_{4}\right)=U_{4}\left(0,1_{n-r}\right) V$ and $\left|U_{4}\right| \neq 0$. Put $\mathfrak{S}_{4}=U_{4}^{-1} C_{4}, \mathfrak{D}_{4}=U_{4}^{-1} D_{4}$; then $\left(\mathfrak{S}_{4}, \mathfrak{D}_{4}\right)$ is primitive and $\mathfrak{C}_{4}{ }^{t} \mathfrak{D}_{4}=U_{4}^{-1} C_{4}{ }^{t} D_{4}{ }^{t} U_{4}^{-1}$ is symmetric. Thus $\left(\mathfrak{S}_{4}, \mathfrak{D}_{4}\right)$ is a symmetric coprime pair. Since $\left(C_{4}, D_{3}, D_{4}\right)$ is primitive, $\left(D_{3}, C_{4}, D_{4}\right)=\left(D_{3}, U_{4}\left(0,1_{n-r}\right) V\right)$ is also primitive. Hence $\left(D_{3}, U_{4}\right)$ is primitive and there is a unimodular matrix $U=\left(\begin{array}{cc}* & * \\ D_{3} & U_{4}\end{array}\right) \in G L(n, Z)$. Put $C=U\left(\begin{array}{cc}0 & 0 \\ 0 & \mathfrak{S}_{4}\end{array}\right), D=U\left(\begin{array}{cc}1_{r} & \\ & \mathfrak{D}_{4}\end{array}\right)$; then $(C, D)$ is a symmetric coprime pair and $\tilde{C}=\left(0, U_{4} \widetilde{E}_{4}\right)=\left(0, C_{4}\right), \tilde{D}=\left(D_{3}, U_{4} \mathfrak{D}_{4}\right)=\left(D_{3}, D_{4}\right)$. This completes the proof.

Q.E.D.

Let $C_{4}, D_{3}, D_{4}$ be those as in Lemma 7 and let $M$ be an element of $\Gamma_{n}$ such that $\tilde{M}=\left(0, C_{4}, D_{3}, D_{4}\right)$. By Lemma 6 there is $K \in \Delta_{n, r}$ such that 
$\left|C_{N}\right| \neq 0,\left(A_{N} C_{N}^{-1}\right)_{1} \equiv 0 \bmod 1$ for $N=K M . \quad$ By Lemma $1 g \tilde{N}=\tilde{M}$ holds for some $g \in G L(n-r, Z)$. Put $P=\left(\begin{array}{c|c}* & 0 \\ \hline 0 & 1_{r_{r}}\end{array}\right) N ;$ then $\tilde{P}=g \tilde{N}=\tilde{M}=$ $\left(0, C_{4}, D_{3}, D_{4}\right),\left|C_{P}\right| \neq 0$, and $\left(A_{P} C_{P}^{-1}\right)_{1} \equiv 0 \bmod 1$. We denote one of such $P$ 's by $M\left[C_{4}, D_{3}, D_{4}\right]$. Then $\Delta_{n, r} M\left[C_{4}, D_{3}, D_{4}\right]$ is uniquely determined by $\left(C_{4}, D_{3}, D_{4}\right)$ by Lemma 1.

Put

$$
\widetilde{\Im}_{n, r}=\left\{\begin{array}{l|l}
\left(C_{4}, D_{3}, D_{4}\right) & \begin{array}{l}
C_{4}, D_{4} \in M_{n-r}(Z), D_{3} \in M_{n-r, r}(Z),\left|C_{4}\right| \neq 0 \\
C_{4}{ }^{t} D_{4}=D_{4}{ }^{t} C_{4} \text { and }\left(C_{4}, D_{3}, D_{4}\right) \text { is primitive }
\end{array}
\end{array}\right\}
$$

For $S, S^{\prime} \in \widetilde{S}_{n, r}$ we define $S \sim S^{\prime}$ by $S^{\prime}=g S$ for some $g \in G L(n-r, Z)$.

LEMMA 8. $\bigcup_{\substack{M \in \Gamma_{n} \\ \operatorname{rank} \tilde{\sigma}_{M}=n-r}} \Delta_{n, r} M=\bigcup \Delta_{n, r} M\left[C_{4}, D_{3}, D_{4}\right]\left(\begin{array}{ll}{ }^{t} U & \\ & U^{-1}\end{array}\right)$, where the right hand is a disjoint union, $\left(C_{4}, D_{3}, D_{4}\right)$ (resp. $\left.{ }^{t} U\right)$ runs over representatives of $\mathfrak{S}_{n, r} / \sim$ (resp. $\left.P_{n, r} \backslash G L(n, Z)\right)$.

Proof. Take an element $M$ of $\Gamma_{n}$ such that $\operatorname{rank} \tilde{C}_{M}=n-r$. Then there exist $g \in G L(n-r, Z), U \in G L(n, Z)$ such that $g^{-1} \tilde{C}_{M}{ }^{t} U^{-1}=\left(0, C_{4}^{(n-r)}\right)$, $\left|C_{4}\right| \neq 0$. Hence $M \in \Delta_{n, r} M\left[C_{4}, D_{3}, D_{4}\right]\left(\begin{array}{c}{ }^{t} U \\ \\ \end{array} U^{-1}\right)$. For $W=\left(\begin{array}{ll}W_{1} & W_{2} \\ & W_{4}\end{array}\right) \in P_{n, r}$

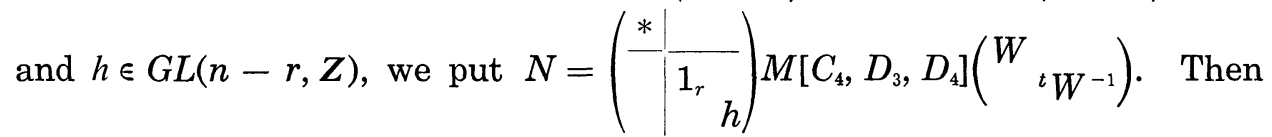
$C_{N}=\left(\begin{array}{cc}* & * \\ 0 & h C_{4} W_{4}\end{array}\right)$ holds. Thus $M$ is contained in some coset $\Delta_{n, r} M\left[C_{4}, D_{3}\right.$, $\left.D_{4}\right]\left(\begin{array}{ll}{ }^{t} U & \\ & U^{-1}\end{array}\right)$ for any specified representatives $\left(C_{4}, D_{3}, D_{4}\right), U$. We must prove the disjointness of the right hand. Suppose $K M\left[C_{4}, D_{3}, D_{4}\right]\left(\begin{array}{ll}{ }^{t} U & \\ & U^{-1}\end{array}\right)$ $=M\left[C_{4}^{\prime}, D_{3}^{\prime}, D_{4}^{\prime}\right]\left(\begin{array}{ll}t & U^{\prime} \\ & U^{\prime-1}\end{array}\right)$ for $\left(C_{4}, D_{3}, D_{4}\right), \quad\left(C_{4}^{\prime}, D_{3}^{\prime}, D_{4}^{\prime}\right) \in \Im_{n, r} / \sim,{ }^{t} U,{ }^{t} U^{\prime} \in$ $P_{n, r} \mid G L(n, Z), K \in \Delta_{n, r}$. There are some $G, G^{\prime} \in \Delta_{n, r}$ such that the first $r$ colums of the $C$-parts of $G M\left[C_{4}, D_{3}, D_{4}\right], G^{\prime} M\left[C_{4}^{\prime}, D_{3}^{\prime}, D_{4}^{\prime}\right]$ vanish as in the proof of Lemma 4. Hence Lemma 5 implies ${ }^{t} U \in P_{n, r}{ }^{t} U^{\prime}$. Thus we have $U=U^{\prime}$ and then $K M\left[C_{4}, D_{3}, D_{4}\right]=M\left[C_{4}^{\prime}, D_{3}^{\prime}, D_{4}^{\prime}\right]$ implies $g^{\prime}\left(C_{4}, D_{3}, D_{4}\right)=$ $\left(C_{4}^{\prime}, D_{3}^{\prime}, D_{4}^{\prime}\right)$ where $g^{\prime}$ is a unimodular matrix defined by the right lower $(n-r) \times(n-r)$ submatrix of $K$. Hence $\left(C_{4}, D_{3}, D_{4}\right)=\left(C_{4}^{\prime}, D_{3}^{\prime}, D_{4}^{\prime}\right)$.

Q.E.D.

We introduce another equivalence relation $\approx$ in $\Im_{n, r}$. For $\left(C_{4}, D_{3}, D_{4}\right)$, 
$\left(C_{4}^{\prime}, D_{3}^{\prime}, D_{4}^{\prime}\right) \in \Im_{n, r}$ we define $\left(C_{4}, D_{3}, D_{4}\right) \approx\left(C_{4}^{\prime}, D_{3}^{\prime}, D_{4}^{\prime}\right)$ by $g\left(C_{4}^{\prime}, D_{3}^{\prime}, D_{4}^{\prime}\right)=$ $\left(C_{4}, D_{3}+C_{4} S_{3}, D_{4}+C_{4} S_{4}\right)$ for some $g \in G L(n-r, Z), S_{3} \in M_{n-r, r}(Z), S_{4}=$ ${ }^{t} S_{4} \in M_{n-r}(Z)$. It is easy to see that $\left(C_{4}^{\prime}, D_{3}^{\prime}, D_{4}^{\prime}\right) \approx\left(C_{4} D_{3}, D_{4}\right)$ if and only if $\Delta_{n, r} M\left[C_{4}^{\prime}, D_{3}^{\prime}, D_{4}^{\prime}\right]=\Delta_{n, r} M\left[C_{4}, D_{3}, D_{4}\right]\left(\frac{1}{0} \mid \frac{S}{1}\right)$ for some $S={ }^{t} S=\left(\begin{array}{ll}0 & S_{2} \\ S_{3} & S_{4}\end{array}\right)$ $\in M_{n}(Z)$.

\section{TheOREM.}

$$
\bigcup_{\substack{M \in \Gamma_{n} n \\
\operatorname{rank} \tilde{C}_{M}=n-r}} \Delta_{n, r} M=\bigcup \Delta_{n, r} M\left[C_{4}, D_{3}, D_{4}\right]\left(\frac{1}{0} \mid \frac{S}{1}\right)\left(\begin{array}{cc}
{ }^{t} U & \\
& U^{-1}
\end{array}\right),
$$

where the right hand is a disjoint union, $\left(C_{4}, D_{3}, D_{4}\right)$ (resp. ${ }^{t} U$ ) runs over representatives of $\widetilde{S}_{n, r} / \approx$ (resp. $P_{n, r} \mid G L(n, Z)$ ) and $S$ runs over $\{S=$ $\left.{ }^{t} S \in M_{n}(Z) \mid S_{1}=0\right\}$.

Proof. This is an immediate corollary of Lemma 8.

Q.E.D.

We remark the following two propositions although they are not used for our aim.

Proposition 1. Take $\left(C_{4}, D_{3}, D_{4}\right) \in \mathfrak{S}_{n, r}$ and $M, N \in \Gamma_{n}$ such that $\tilde{M}=$ $\tilde{N}=\left(0, C_{4}, D_{3}, D_{4}\right),\left|C_{M}\right|\left|C_{N}\right| \neq 0\left(A_{M} C_{M}^{-1}\right)_{1} \equiv\left(A_{N} C_{N}^{-1}\right)_{1} \equiv 0 \bmod 1$. Then we have $G L(r, Z)\left(C_{M}\right)_{1}=G L(r, Z)\left(C_{N}\right)_{1}$.

Proof. Put $A_{M}=\left(\begin{array}{ll}A_{1} & A_{2} \\ A_{3} & A_{4}\end{array}\right), C_{M}=\left(\begin{array}{ll}C_{1} & C_{2} \\ & C_{4}\end{array}\right) ;{ }^{t} A_{M} C_{M}={ }^{t} C_{M} A_{M}$ implies ${ }^{t} A_{1} C_{1}={ }^{t} C_{1} A_{1}$ and $A_{1} C_{1}^{-1}={ }^{t}\left(A_{1} C_{1}^{-1}\right)$ is an integral matrix. Put $K=$ $\left(\begin{array}{ll|ll}0 & 0 & -1_{r} & 0 \\ 0 & 1_{n-r} & 0 & 0 \\ \hline 1_{r} & 0 & -A_{1} C_{1}^{-1} & 0 \\ 0 & 0 & 0 & 1_{n-r}\end{array}\right)$; then $K \in \Delta_{n, r}$ and $C_{K M}=\left(\begin{array}{ll}0 & * \\ 0 & C_{4}\end{array}\right)$. We define similarly $K^{\prime} \in \Delta_{n, r}$ for $N$. Then $\Delta_{n, r} K M=\Delta_{n, r} K^{\prime} N$ and Lemma 5 imply

$$
K^{\prime} N=\left(\begin{array}{cc}
U & U S \\
& { }^{t} U^{-1}
\end{array}\right) K M,
$$

where $\left(\begin{array}{ll}U & U S \\ & { }^{t} U^{-1}\end{array}\right) \in \Delta_{n, r}, U=\left(\begin{array}{ll}U_{1}^{(r)} & \\ U_{3} & U_{4}\end{array}\right) \in G L(n, Z)$. Then $\left(A_{K^{\prime} N}\right)_{1}=\left(U A_{K M}\right.$ $\left.+U S C_{K M}\right)_{1}$ implies $-\left(C_{N}\right)_{1}=-U_{1}\left(C_{M}\right)_{1}$. Thus we have $G L(r, Z)\left(C_{M}\right)_{1}=$ $G L(r, Z)\left(C_{N}\right)_{1}$. Q.E.D.

Let $\left(C_{4}, D_{3}, D_{4}\right)$ be an element of $\widetilde{S}_{n, r}$ and define matrices $U=\left(\begin{array}{ll}U_{1} & U_{2} \\ U_{3} & U_{4}\end{array}\right)$, $\left(\mathfrak{\Im}_{4}, \mathfrak{D}_{4}\right)$ and $(C, D)$ as in the proof of Lemma 7 ; then $C_{4}=U_{4} \mathfrak{\Im}_{4}, D_{4}=U_{4} \mathfrak{D}_{4}$ 
and $D_{3}=U_{3}$. Taking, $\mathfrak{N}_{4}, \mathfrak{B}_{4}$ such that $\left(\begin{array}{ll}\mathfrak{U}_{4} & \mathfrak{B}_{4} \\ \mathfrak{S}_{4} & \mathfrak{D}_{4}\end{array}\right) \in \Gamma_{n-r}$ we put

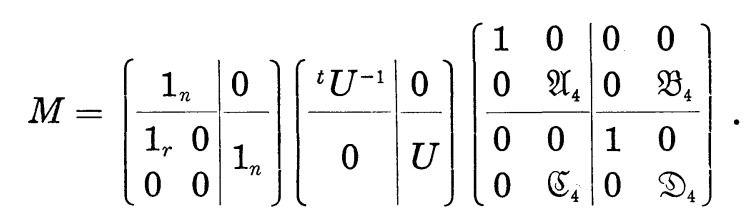

Then $C_{4}=U_{4} \Im_{4}$ and $\left|C_{4}\right| \neq 0$ imply $\left|U_{4}\right| \neq 0$ and $\left|U_{1}-U_{2} U_{4}^{-1} U_{3}\right| \neq 0$ follows from

$$
\left(\begin{array}{cc}
1 & -U_{2} U_{4}^{-1} \\
0 & 1
\end{array}\right) U=\left(\begin{array}{cc}
U_{1}-U_{2} U_{4}^{-1} U_{3} & 0 \\
* & *
\end{array}\right)
$$

Hence we have

$$
U^{-1}=\left(\begin{array}{cc}
\left(U_{1}-U_{2} U_{4}^{-1} U_{3}\right)^{-1} & -\left(U_{1}-U_{2} U_{4}^{-1} U_{3}\right)^{-1} U_{2} U_{4}^{-1} \\
-U_{4}^{-1} U_{3}\left(U_{1}-U_{2} U_{4}^{-1} U_{3}\right)^{-1} & U_{4}^{-1}+U_{4}^{-1} U_{3}\left(U_{1}-U_{2} U_{4}^{-1} U_{3}\right)^{-1} U_{2} U_{4}^{-1}
\end{array}\right)
$$

Putting ${ }^{t} U^{-1}=V$, we have

$$
\begin{array}{rlrl}
A_{M} & =\left(\begin{array}{ll}
V_{1} & V_{2} \mathfrak{U}_{4} \\
V_{3} & V_{4} \mathfrak{U}_{4}
\end{array}\right), & B_{M}=\left(\begin{array}{ll}
0 & * \\
0 & *
\end{array}\right), \\
C_{M}=\left(\begin{array}{cc}
V_{1} & V_{2} \mathfrak{U}_{4}+U_{2} \mathfrak{S}_{4} \\
0 & C_{4}
\end{array}\right), & D_{M}=\left(\begin{array}{cc}
U_{1} & V_{2} \mathfrak{B}_{4}+U_{2} \mathfrak{I}_{4} \\
D_{3} & D_{4}
\end{array}\right),
\end{array}
$$

For this special extension $M=\left(\begin{array}{ll}A & B \\ C & D\end{array}\right) \in \Gamma_{n}$ of $\left(0, C_{4}, D_{3}, D_{4}\right)$ we have

Lemma 8. $X=\left(B_{1}-A_{1} C_{1}^{-1} D_{1}-A_{2} C_{4}^{-1} D_{3}+A_{1} C_{1}^{-1} C_{2} C_{4}^{-1} D_{3}\right)^{t} C_{1}$ is integral.

$$
\text { Proof. } \begin{aligned}
X & =\left(-U_{1}-V_{2} \mathfrak{U}_{4} C_{4}^{-1} D_{3}+\left(V_{2} \mathfrak{U}_{4}+U_{2} \mathfrak{S}_{4}\right) C_{4}^{-1} D_{3}\right)^{t} V_{1} \\
& =\left(-U_{1}+U_{2} \mathfrak{S}_{4} C_{4}^{-1} D_{3}\right)^{t} V_{1}=-1_{r} .
\end{aligned}
$$

Proposition 2. Take $N=M\left[C_{4}, D_{3}, D_{4}\right]$ for $\left(C_{4}, D_{3}, D_{4}\right) \in \mathfrak{S}_{n, r}$ and a half-integral symmetric matrix $P^{(n)}$ such that ${ }^{t}\left(C_{N}\right)_{1}^{-1} P_{1}\left(C_{N}\right)_{1}^{-1}$ is half-integral. Then $\sigma\left(P C_{N}^{-1} D_{N}\right) \bmod Z$ is uniquely determined by $\left(C_{4}, D_{3}, D_{4}\right)$ and $P$.

Proof. Take $M \in \Gamma_{n}$ such that $\tilde{M}=\left(0, C_{4}, D_{3}, D_{4}\right)\left|C_{M}\right| \neq 0,\left(A_{M} C_{M}^{-1}\right)_{1} \equiv$ $0 \bmod 1$ and put $C_{M}=C, D_{M}=D$; then we have

$$
C^{-1} D=\left(\begin{array}{cc}
C_{1}^{-1}\left(D_{1}-C_{2} C_{4}^{-1} D_{3}\right) & { }^{t}\left(C_{4}^{-1} D_{3}\right) \\
C_{4}^{-1} D_{3} & C_{4}^{-1} D_{4}
\end{array}\right)
$$

and $\left(C^{-1} D\right)_{2},\left(C^{-1} D\right)_{3},\left(C^{-1} D\right)_{4}$ are only dependent of $\left(C_{4}, D_{3}, D_{4}\right)$. Take any 
extension $N=M\left[C_{4}, D_{3}, D_{4}\right]$ and define $K, K^{\prime}, U, S$ as in the proof of Proposition 1; then $\tilde{C}_{N}=\tilde{C}_{M}$ implies $U_{4}=1_{n-r}$ and we have

$$
\begin{aligned}
& C_{N}=\left(\begin{array}{cc}
-U_{1} S_{1} & 0 \\
0 & 0
\end{array}\right) A_{M}+\left(\begin{array}{cc}
U_{1}+U_{1} S_{1} A_{1} C_{1}^{-1} & -U_{1} S_{2} \\
0 & 1
\end{array}\right) C_{M} \\
& D_{N}=\left(\begin{array}{cc}
-U_{1} S_{1} & 0 \\
0 & 0
\end{array}\right) B_{M}+\left(\begin{array}{cc}
U_{1}+U_{1} S_{1} A_{1} C_{1}^{-1} & -U_{1} S_{2} \\
0 & 1
\end{array}\right) D_{M} .
\end{aligned}
$$

Hence $\left(C_{M}^{-1} D_{M}-C_{N}^{-1} D_{N}\right)_{1}=C_{1}^{-1} S_{1}\left(B_{1}-A_{1} C_{1}^{-1} D_{1}-A_{2} C_{4}^{-1} D_{3}+A_{1} C_{1}^{-1} C_{2} C_{4}^{-1} D_{3}\right)$. Now we suppose that $M$ is a special extension in Lemma 8; then $C_{1}\left(C_{M}^{-1} D_{M}\right.$ $\left.-C_{N}^{-1} D_{N}\right)^{t} C_{1} \equiv 0 \bmod 1$. Therefore $\sigma\left(P C_{M}^{-1} D_{M}\right)-\sigma\left(P C_{N}^{-1} D_{N}\right)=\sigma\left(P_{1}\left(C_{M}^{-1} D_{M}\right.\right.$ $\left.\left.-C_{N}^{-1} D_{N}\right)_{1}\right)=\sigma\left({ }^{t} C_{1}^{-1} P_{1} C_{1}^{-1}\left(C_{1}\left(C_{M}^{-1} D_{M}-C_{N}^{-1} D_{N}\right)^{t} C_{1}\right)\right) \equiv 0 \bmod 1 . \quad$ Q.E.D.

\section{$\S 2$.}

Through this section we fix natural numbers $r, n, k$ such that $1 \leq r$ $\leq n-1, k \geq n+r+2, k \equiv 0 \bmod 2$. Denote by $f$ a cusp form of degree $r$ and weight $k$ which is also fixed.

For $M \in \Gamma_{n}$ we put

$$
\left.(f \mid M)(Z)=f(M\langle Z\rangle)_{1}\right)\left|C_{M} Z+D_{M}\right|^{-k},
$$

where $M\langle Z\rangle=\left(A_{M} Z+B_{M}\right)\left(C_{M} Z+D_{M}\right)^{-1},\left(Z \in H_{n}\right)$, and $(M\langle Z\rangle)_{1}$ is the upper left $r \times r$ submatrix of $M\langle Z\rangle$ as in $\S 1$, and $(f \mid M)(Z)=f\left(M\langle Z\rangle^{*}\right) M\{Z\}^{-k}$ in the notation of Klingen [3]. It is easy to see that $(f \mid M)(Z)=(f \mid N M)(Z)$ for any $N \in \Delta_{n, r}$.

Put $E_{1}(Z)=\sum_{M}(f \mid M)(Z)$ (resp. $E_{2}(Z)=\sum_{M}(f \mid M)(Z)$ ) where $M$ runs over representatives of $\Delta_{n, r} \backslash \Gamma_{n}$ such that $\operatorname{rank} \tilde{C}_{M}=n-r$ (resp. $\operatorname{rank} \tilde{C}_{M}$ $<n-r)$.

LEMMA 1. Let $N$ be an element of $\Gamma_{n}$ such that rank $\tilde{C}_{N}<n-r$ and put $\frac{\partial}{\partial Y}=\left(\frac{1}{2}\left(1+\delta_{i j}\right) \frac{\partial}{\partial y_{i j}}\right)$. Then we have $\left|\frac{\partial}{\partial Y}\right|(f \mid N)(Z)=0$ where $Y$ $=\operatorname{Im} Z$.

Proof. By Lemma 2 in $\S 1$ there exist $M \in \Delta_{n, n-1}, U \in G L(n, Z)$ such that $\Delta_{n, r} N \ni M\left(\begin{array}{ll}U & \\ & { }^{t} U^{-1}\end{array}\right)$. Put $A_{M}=\left(\begin{array}{ll}A_{1} & 0 \\ A_{3} & A_{4}\end{array}\right), B_{M}=\left(\begin{array}{ll}B_{1} & B_{2} \\ B_{3} & B_{4}\end{array}\right), C_{M}=\left(\begin{array}{ll}C_{1} & 0 \\ 0 & 0\end{array}\right), D_{M}$ $=\left(\begin{array}{ll}D_{1} & D_{2} \\ 0 & D_{4}\end{array}\right)$ and $Z=\left(\begin{array}{ll}Z_{1} & Z_{2} \\ { }^{t} Z_{2} & Z_{4}\end{array}\right)$ where $A_{1}, \cdots, D_{1}$ and $Z_{1}$ are $(n-1) \times$ $(n-1)$ matrices. Then we have $C_{M} Z+D_{M}=\left(\begin{array}{cc}C_{1} Z_{1}+D_{1} & * \\ 0 & D_{4}\end{array}\right), M\langle Z\rangle=$ $\left(\begin{array}{c}\left(A_{1} Z_{1}+B_{1}\right)\left(C_{1} Z_{1}+D_{1}\right)^{-1} \\ * \\ *\end{array}\right)$. Hence $(f \mid M)(Z)$ does not depend on $Z_{2}, Z_{4}$ 
and so $|\partial / \partial Y|(f \mid M)(Z)=0$. Since $(f \mid N)(Z)=(f \mid M)\left(U Z^{t} U\right)$ and $|Y||\partial / \partial Y|$ is invariant under the transformation $Y \rightarrow U Y^{t} U$ on $\left\{Y^{(n)} \mid Y>0\right\}$, we have

$$
\begin{aligned}
& |Y|\left|\frac{\partial}{\partial Y}\right|(f \mid N)(Z)=|Y|\left|\frac{\partial}{\partial Y}\right|(f \mid M)\left(U Z^{t} U\right) \\
& \quad=\left.|Y|\left|\frac{\partial}{\partial Y}\right|(f \mid M)\left(U X^{t} U+i Y\right)\right|_{Y \rightarrow U Y^{t} U}=0,
\end{aligned}
$$

where $Z=X+i Y$.

Q.E.D.

Proposition 1. $\mathrm{E}_{2}(Z)$ has Fourier expansion $\sum_{T} a_{2}(T) e(\sigma(T Z))$ such that $a_{2}(T)=0$ for $T>0$.

Proof. Put $N=M\left(\frac{1}{\mid} \mid \frac{S}{1}\right)$ where $N, M \in \Gamma_{n},{ }^{t} S=S \in M_{n}(Z)$. Then $\operatorname{rank} \tilde{C}_{N}=\operatorname{rank} \tilde{C}_{M}$. Hence $E_{2}(Z+S)=E_{2}(Z)$ for any $S={ }^{t} S \in M_{n}(Z)$. Thus $E_{2}(Z)$ has Fourier expansion $\sum_{T} a_{2}(T) e(\sigma(T Z))$. From Lemma 1 follows

$$
\left|\frac{\partial}{\partial Y}\right| E_{2}(Z)=\sum_{T} a_{2}(T)|-2 \pi T| e(\sigma(T Z))=0 .
$$

Hence $a_{2}(T)$ vanishes if $T$ is positive definite.

Q.E.D.

For a natural number $m$ we put

$$
\begin{aligned}
& \Lambda_{m}=\left\{S \in M_{m}(Z) \mid S={ }^{t} S\right\}, \\
& \Lambda_{m}^{*}=\left\{S \in M_{m}(\boldsymbol{Q}) \mid S={ }^{t} S: \text { half-integral }\right\} .
\end{aligned}
$$

$\Lambda_{m}^{*}$ is the dual lattice of $\Lambda_{m}$ via $\sigma\left(S S^{\prime}\right)$.

The following is well known ([1], [5], [8]).

Lemma 2. For a positive definite matrix $Y^{(m)}$ and $\rho>m+1$,

$$
\sum_{F \in\lrcorner_{m}}|Y+2 \pi i F|^{-\rho}
$$

is absolutely convergent and

$$
\Gamma_{m}(\rho) \sum_{F \in A_{m}}|Y+2 \pi i F|^{-\rho}=2^{-m(m-1) / 2} \sum_{\substack{T>0 \\ T \in \Lambda_{m}^{*}}}|T|^{\rho-(m+1) / 2} e^{-\sigma(T Y)},
$$

where $\Gamma_{m}(\rho)=\pi^{m(m-1) / 4} \prod_{\nu=0}^{m-1} \Gamma(\rho-\nu / 2)$.

Lemma 3. For a positive number a,

$$
\begin{aligned}
& \sum_{F \in \Lambda_{m}}\left|(2 \pi i)^{-1}(Z+a F)\right|^{-\rho} \\
& \quad=2^{-m(m-1) / 2}(2 \pi)^{2 m \rho} \Gamma_{m}(\rho)^{-1} a^{-m \rho} \sum_{\substack{T>0 \\
T \in A_{m}^{*}}}|T|^{\rho-(m+1) / 2} e\left(a^{-1} \sigma(T Z)\right),
\end{aligned}
$$


where $Z \in H_{m}$ and $\rho>m+1$.

Proof. This is an immediate corollary of Lemmal 2.

LEMmA 4. If $a, b$ are complex numbers such that $\operatorname{Re} a>0$, then we have

$$
\int_{R} \exp \left(-a x^{2}+2 b x\right) d x=\sqrt{\pi / a} \exp \left(b^{2} / a\right),
$$

where $\sqrt{\pi / a}$ is real positive if $a$ is real.

Proof. This is also well known.

The following is an easy generalization.

Lemma 5. If $A$ is a symmetric matrix of $M_{m}(C)$ such that $\operatorname{Re} A>0$ and $b$ is an element of $C^{m}$, then we have

$$
\int_{R^{m}} \exp \left(-{ }^{t} x A x+2^{t} b x\right) d x=\sqrt{\operatorname{det}\left(\pi A^{-1}\right)} \exp \left({ }^{t} b A^{-1} b\right),
$$

where $\sqrt{\operatorname{det}\left(\pi A^{-1}\right)}$ is real positive if $A$ is real.

We need the following generalization.

Lemma 6. If $A$ is a symmetric matrix of $M_{n-r}(C)$ such that $\operatorname{Re} A>0$ and $W_{1}^{(r)}>0$ and $Q$ is an element of $M_{n-r, r}(C)$, then we have

$$
\begin{aligned}
& \int_{X \in M_{r, n-r}(R)} \exp \left(-2 \pi \sigma\left(W_{1} X A^{t} X\right)+2 \pi \sigma(X Q)\right) d X \\
& =\left|W_{1}\right|^{((r-n) / 2} 2^{r(r-n) / 2} \sqrt{\left(\operatorname{det} A^{-1}\right)^{r}} \exp \left(\frac{\pi}{2} \sigma\left({ }^{t} Q A^{-1} Q W_{1}^{-1}\right)\right),
\end{aligned}
$$

where $\sqrt{\left(\operatorname{det} A^{-1}\right)^{r}}$ is real positive if $A$ real.

Proof. Put ${ }^{t} X=\left({ }^{t} x_{1}, \cdots,{ }^{t} x_{r}\right)$ and ${ }^{t} x=\left(x_{1}, \cdots, x_{r}\right) \in M_{1,(n-r) r}(R)$. Then we have $\sigma\left(X A^{t} X\right)={ }^{t} x\left(\begin{array}{llll}A & & \\ & \ddots & \\ & & \\ & & A\end{array}\right) x$ where $r A^{\prime}$ 's are on the diagonal. Denoting $Q W^{-1}$ by $\left(y_{1}, \cdots, y_{r}\right)$ where $W=\sqrt{W_{1}}>0$, we have $\sigma\left(X Q W^{-1}\right)=$ $\left({ }^{t} y_{1}, \cdots,{ }^{t} y_{r}\right) x$. Thus the integral of the left side is

$$
\begin{aligned}
& |W|^{r-n} \int_{X \in M_{r, n-r(R)}} \exp \left(-2 \pi \sigma\left(X A^{t} X\right)+2 \pi \sigma\left(X Q W^{-1}\right)\right) d X \\
& \quad=\left|W_{1}\right|^{(r-n) / 2} \int_{R^{(n-r) r}} \exp \left(-2 \pi^{t} x\left(^{A} \cdot{ }_{A}\right) x+2 \pi\left({ }^{t} y_{1}, \ldots,{ }^{t} y_{r}\right) x\right) d x
\end{aligned}
$$




$$
\left.=\left|W_{1}\right|^{(r-n) / 2} 2^{r(r-n) / 2} \sqrt{\left(\operatorname{det} A^{-1}\right)^{r}} \exp \left(\frac{\pi}{2} \sigma{ }^{t} W^{-1} t Q A^{-1} Q W^{-1}\right)\right) . \quad \text { Q.E.D. }
$$

LEMma 7. If $N$ is an element of $\Gamma_{n}$ such that $\left|C_{N}\right| \neq 0,\left(A_{N} C_{N}^{-1}\right)_{1} \equiv$ $0 \bmod 1$, then we have

$$
(f \mid N)(Z)=\left|C_{N}\right|^{k}\left|W_{4}\right|^{-k} f\left(W_{1}-W_{2} W_{4}^{-1 t} W_{2}\right),
$$

where

$$
W=\left(\begin{array}{ll}
W_{1}^{(r)} & W_{2} \\
{ }^{t} W_{2} & W_{4}
\end{array}\right)=C_{N} Z^{t} C_{N}+D_{N}{ }^{t} C_{N}
$$

Proof. Put $N=\left(\begin{array}{ll}A & B \\ C & D\end{array}\right)$. Since $B-A C^{-1} D=B-A^{t}\left(C^{-1} D\right)=\left(B^{t} C\right.$ $\left.-A^{t} D\right){ }^{t} C^{-1}=-{ }^{t} C^{-1}$, we have $N\langle Z\rangle=(A Z+B)(C Z+D)^{-1}=A C^{-1}-$ ${ }^{t} C^{-1}(C Z+D)^{-1}=A C^{-1}-W^{-1}$. From the identity

$$
W=\left(\begin{array}{cc}
1 & W_{2} W_{4}^{-1} \\
0 & 1
\end{array}\right)\left(\begin{array}{cc}
W_{1}-W_{2} W_{4}^{-1 t} W_{2} & 0 \\
0 & W_{4}
\end{array}\right)\left(\begin{array}{cc}
1 & 0 \\
W_{4}^{-1 t} W_{2} & 1
\end{array}\right)
$$

follows

$$
W^{-1}=\left(\begin{array}{cc}
\left(W_{1}-W_{2} W_{4}^{-1}{ }^{t} W_{2}\right)^{-1} & * \\
* & *
\end{array}\right)
$$

Hence we have

$$
\begin{aligned}
(f \mid N)(Z) & =f\left(-\left(W_{1}-W_{2} W_{4}^{-1 t} W_{2}\right)^{-1}\right)\left|W^{t} C^{-1}\right|^{-k} \\
& =f\left(W_{1}-W_{2} W_{4}^{-1}{ }^{t} W_{2}\right)\left|W_{1}-W_{2} W_{4}^{-1 t}{ }^{t} W_{2}\right|^{k}|W|^{-k}|C|^{k} \\
& =|C|^{k}\left|W_{4}\right|^{-k} f\left(W_{1}-W_{2} W_{4}^{-1 t} W_{2}\right) .
\end{aligned}
$$

Let $N=\left(\begin{array}{ll}A & B \\ C & D\end{array}\right)$ be an element of $\Gamma_{n}$ such that $|C| \neq 0,\left(A C^{-1}\right)_{1} \equiv$ $0 \bmod 1, C_{3}=0$ where we decompose $M \in M_{n}(C)$ as $M=\left(\begin{array}{ll}M_{1}^{(r)} & M_{2} \\ M_{3} & M_{4}\end{array}\right)$ as in $\S 1$ and take a natural number $p$ such that $p C^{-1}$ is an integral matrix. We fix $N, p$ till Lemma 13 . Now we calculate

$$
S=\left(\sum_{\left.t_{S_{2}}^{0} S_{S_{4}}\right) \in p^{2} A_{n}}\left(f \mid N\left(\frac{1}{0} \mid \frac{C^{-1} S^{t} C^{-1}}{1}\right)\right)(Z) .\right.
$$

Put $W=C Z^{t} C+D^{t} C$. For $S=\left(\begin{array}{cc}0 & S_{2} \\ { }^{t} S_{2} & S_{4}\end{array}\right) \in \Lambda_{n}$ we have

$$
N\left(\frac{1}{0} \mid \frac{C^{-1} S^{t} C^{-1}}{1}\right)=\left(\frac{*}{C} \mid \frac{*}{S^{t} C^{-1}+D}\right)
$$


and $C Z^{t} C+\left(S^{t} C^{-1}+D\right)^{t} C=W+S$. If, moreover, $S \in p^{2} \Lambda_{n}$, then

$$
\begin{aligned}
& \left(f \mid N\left(\frac{1}{0} \mid \frac{C^{-1} S^{t} C^{-1}}{1}\right)\right)(Z) \\
& \quad=|C|^{k}\left|W_{4}+S_{4}\right|^{-k} f\left(W_{1}-\left(W_{1}+S_{2}\right)\left(W_{4}+S_{4}\right)^{-1}{ }^{t}\left(W_{2}+S_{2}\right)\right) .
\end{aligned}
$$

First we calculate, for $t>0, t \in \Lambda_{r}^{*}$,

$$
\sum_{S_{2} \in p^{2} M_{r, n-r}(\boldsymbol{Z})} e\left(-\sigma\left(t\left(W_{2}+S_{2}\right) W_{4}^{-1}{ }^{t}\left(W_{2}+S_{2}\right)\right)\right) .
$$

It equals

$$
\begin{aligned}
& \sum_{S_{2} \in M_{r, n-r}(\boldsymbol{Z})} e\left(-\sigma\left(t\left(W_{2}+p^{2} S_{2}\right) W_{4}^{-1}{ }^{t}\left(W_{2}+p^{2} S_{2}\right)\right)\right) \\
& =\sum_{S_{2} \in M_{r, n-r}(\boldsymbol{Z})} \int_{X \in M_{r, n-r}(\boldsymbol{R})} e\left(-\sigma\left(t\left(W_{2}+p^{2} X\right) W_{4}^{-1}{ }^{t}\left(W_{2}+p^{2} X\right)\right)\right) e\left(\sigma\left(S_{2}{ }^{t} X\right)\right) d X \\
& =e\left(-\sigma\left(t W_{2} W_{4}^{-1}{ }^{t} W_{2}\right)\right) \sum_{S_{2} \in M_{r, n-r}(Z)} \int_{X \in M_{r, n-r}(\boldsymbol{R})} \exp \left(-2 \pi \sigma\left(p^{4} t X\left(i W_{4}^{-1}\right)^{t} X\right)\right) \\
& \quad \times \exp \left(2 \pi \sigma\left(X\left(-2 i p^{2} W_{4}^{-1}{ }^{t} W_{2} t+i^{t} S_{2}\right)\right)\right) d X \\
& =e\left(-\sigma\left(t W_{2} W_{4}^{-1{ }^{t} t} W_{2}\right)\right) \sum_{S_{2} \in M_{r, n-r}(Z)}\left|p^{4} t\right|^{(r-n) / 2} 2^{r(r-n) / 2} \sqrt{\operatorname{det}\left(i^{-1} W_{4}\right)^{r}} \\
& \times \exp \left((\pi / 2) \sigma\left({ }^{t} Q\left(i^{-1} W_{4}\right) Q p^{-4} t^{-1}\right)\right),
\end{aligned}
$$

where we put $Q=-2 i p^{2} W_{4}^{-1}{ }^{t} W_{2} t+i^{t} S_{2}$.

Since

$$
\begin{aligned}
& \sigma\left({ }^{t} Q\left(i^{-1} W_{4}\right) Q p^{-4} t^{-1}\right) \\
& \quad=p^{-4} i \sigma\left(4 p^{4} W_{2} W_{4}^{-1}{ }^{t} W_{2} t-4 p^{2} W_{2}{ }^{t} S_{2}+S_{2} W_{4}{ }^{t} S_{2} t^{-1}\right),
\end{aligned}
$$

we have

$$
\begin{aligned}
& \sum_{S_{2} \in p^{2} M_{r, n-r}(Z)} e\left(-\sigma\left(t\left(W_{2}+S_{2}\right) W_{4}^{-1} t\left(W_{2}+S_{2}\right)\right)\right) \\
&= 2^{r(r-n) / 2} \cdot p^{2 r(r-n)}|t|^{(r-n) / 2} \sqrt{\operatorname{det}\left(i^{-1} W_{4}\right)^{r}} \\
& \times \sum_{S_{2} \in M_{r, n-r}(Z)} e\left(\left(4 p^{4}\right)^{-1} \sigma\left(-4 p^{2} W_{2}^{t} S_{2}+S_{2} W_{2}^{t} S_{2} t^{-1}\right)\right) .
\end{aligned}
$$

Put $f\left(z^{(r)}\right)=\sum_{t \in \Lambda_{r}^{*}}^{t>0} b(t) e(\sigma(t z))$; then $b(t)=O\left(|t|^{k / 2}\right)$ is known [7].

\section{LEMMA 8.}

$$
\begin{aligned}
\sum_{S=\left(\begin{array}{c}
0 \\
t_{S_{2}} \\
S_{0} \\
0
\end{array}\right) \in p^{2 A_{n}}}\left(f \mid N\left(\frac{1}{0} \mid \frac{C^{-1} S^{t} C^{-1}}{1}\right)\right)(Z) \\
=|C|^{k} 2^{r(r-n) / 2} p^{2 r(r-n)}\left|W_{4}\right|^{-k} \sqrt{\operatorname{det}\left(i^{-1} W_{4}\right)^{r}} \\
\quad \times \sum_{\substack{t>0 \\
t \in A_{r}^{*} \\
S_{2} \in M_{r, n-r}(Z)}}|t|^{(r-n) / 2} b(t) e\left(\sigma\left(t W_{1}\right)+\left(4 p^{4}\right)^{-1} \sigma\left(-4 p^{2} W_{2}{ }^{t} S_{2}+S_{2} W_{4}{ }^{t} S_{2} t^{-1}\right)\right)
\end{aligned}
$$


where the right hand is absolutely convergent.

Proof. Define a matrix $P \in M_{r}(R)$ by

$$
P=\operatorname{Im}\left(W_{1}-\left(W_{2}+X\right) W_{4}^{-1} t\left(W_{2}+X\right)\right)-(X+Q) \operatorname{Im}\left(-W_{4}^{-1}\right)^{t}(X+Q),
$$

where $Q=\operatorname{Re} W_{2}+\operatorname{Im} W_{2} \cdot \operatorname{Re}\left(-W_{4}^{-1}\right)\left(\operatorname{Im}\left(-W_{4}^{-1}\right)\right)^{-1}, X \in M_{r, n-r}(R)$. Then $P$ is independent of $X$. Since $\left(W_{1}-\left(W_{2}+X\right) W_{4}^{-1}\left(W_{2}+X\right)\right)^{-1}$ is the upper left $r \times r$ matrix of

$$
\left(W+\left(\begin{array}{ll}
0 & X \\
{ }^{t} X & 0
\end{array}\right)\right)^{-1}
$$

we have $W_{1}-\left(W_{2}+X\right) W_{4}^{-1 t}\left(W_{2}+X\right) \in H_{r}$ and its imaginary part is positive definite. Hence, putting $X=-Q$, we see that $P$ is positive definite. Now we have

$$
\begin{aligned}
& \left|e\left(\sigma\left(t\left(W_{1}-\left(W_{2}+S_{2}\right) W_{4}^{-1}{ }^{t}\left(W_{2}+S_{2}\right)\right)\right)\right)\right| \\
& =\exp \left(-2 \pi \sigma\left(t P+t\left(S_{2}+Q\right) \operatorname{Im}\left(-W_{4}^{-1}\right)^{t}\left(S_{2}+Q\right)\right)\right) \\
& \quad<\exp \left(-2 \pi \varepsilon \sigma\left(t+\left(S_{2}+Q\right)^{t}\left(S_{2}+Q\right)\right)\right)
\end{aligned}
$$

where $\varepsilon>0$ is defined by

$$
P>\varepsilon 1_{r}, \quad \operatorname{Im}\left(-W_{4}^{-1}\right)>\sqrt{\varepsilon} 1_{n-r}, \quad t>\sqrt{\varepsilon} 1_{r} \quad \text { for } t \in \Lambda_{r}^{*}, t>0 .
$$

Then it is easy to see that

$$
\sum_{\substack{t>0 \\ t \in A_{r}^{*} \\ S_{2} \in p^{2} M_{r}, n-r}} b(t) e\left(\sigma\left(t\left(W_{1}-\left(W_{2}+S_{2}\right) W_{4}^{-1}{ }^{t}\left(W_{2}+S_{2}\right)\right)\right)\right.
$$

is absolutely convergent.

To prove that the right hand is absolutely convergent, it is enough to show

$$
\begin{gathered}
\sum_{S_{2} \in M r, n-r(Z)}\left|e\left(\sigma\left(t W_{1}\right)+\left(4 p^{4}\right)^{-1} \sigma\left(-4 p^{2} W_{2}{ }^{t} S_{2}+S_{2} W_{4}{ }^{t} S_{2} t^{-1}\right)\right)\right| \\
=O\left(|t|^{n-r} \exp (-2 \pi \varepsilon \sigma(t))\right) \quad \text { for some } \varepsilon>0 .
\end{gathered}
$$

$\operatorname{Im}\left(\sigma\left(t W_{1}\right)+\left(4 p^{4}\right)^{-1} \sigma\left(-4 p^{2} W_{2}^{t} S_{2}+S_{2} W_{4}^{t} S_{2} t^{-1}\right)\right)$ is equal to

$$
\begin{aligned}
& \sigma\left((\operatorname{Im} W)\left(\begin{array}{ccc}
t & -\left(2 p^{2}\right)^{-1} S_{2} \\
-\left(2 p^{2}\right)^{-1}{ }^{t} S_{2} & \left(4 p^{4}\right)^{-1}{ }^{t} S_{2} t^{-1} S_{2}
\end{array}\right)\right) \\
& \quad=\sigma\left(\left(\begin{array}{ccc}
1 & -\left(2 p^{2}\right)^{-1} t^{-1} S_{2} \\
0 & 1 & 1
\end{array}\right)(\operatorname{Im} W)\left(\begin{array}{cc}
1 & 0 \\
\left(-\left(2 p^{2}\right)^{-1}{ }^{t} S_{2} t^{-1}\right. & 1
\end{array}\right)\left(\begin{array}{ll}
t & 0 \\
0 & 0
\end{array}\right)\right)
\end{aligned}
$$


and, taking a positive number $\varepsilon_{1}$ such that $\operatorname{Im} W>\varepsilon_{1} 1_{n}$, we have

$$
\begin{aligned}
& \left|e\left(\sigma\left(t W_{1}\right)+\left(4 p^{4}\right)^{-1} \sigma\left(-4 p^{2} W_{2}{ }^{t} S_{2}+S_{2} W_{4}{ }^{t} S_{2} t^{-1}\right)\right)\right| \\
& \leq \exp \left(-2 \pi \varepsilon_{1} \sigma\left(t+\left(4 p^{4}\right)^{-1} S_{2}{ }^{t} S_{2} t^{-1}\right)\right)
\end{aligned}
$$

Hence we have only to prove that, for $\varepsilon^{\prime}=2 \pi \varepsilon_{1}\left(4 p^{4}\right)^{-1}$,

$$
\sum_{S_{2} \in M_{r, n_{-r}(Z)}} \exp \left(-\varepsilon^{\prime} \sigma\left(S_{2}{ }^{t} S_{2} t^{-1}\right)\right)=O\left(|t|^{n-r}\right) \quad \text { for } t>0, t \in \Lambda_{r}^{*}
$$

Without loss of generality we may assume that $t^{-1}$ is in some Siegel domain. Then there are positive constants $\varepsilon_{2}, \varepsilon_{3}$ such that

$$
\varepsilon_{2} \delta<t^{-1}<\varepsilon_{3} \delta
$$

where $\delta$ is a diagonal matrix defined by

$$
\delta=\left(\begin{array}{ccc}
\delta_{1} & & 0 \\
& \ddots & \\
0 & & \delta_{r}
\end{array}\right)={ }^{t} T^{-1} t^{-1} T^{-1}, \quad T=\left(\begin{array}{lll}
1 & & * \\
& \ddots & \\
0 & & 1
\end{array}\right)
$$

Then $t<\varepsilon_{2}^{-1} \delta^{-1}$ and $t \in \Lambda_{r}^{*}$ imply that $\delta_{i}<\varepsilon_{2}^{-1}$. Therefore we have

$$
\begin{aligned}
& \sum_{S_{2} \in M_{r, n-r}(\boldsymbol{Z})} \exp \left(-\varepsilon^{\prime} \sigma\left(S_{2}{ }^{t} S_{2} t^{-1}\right)\right)<\sum_{S_{2} \in M_{r, n-r}(Z)} \exp \left(-\varepsilon^{\prime} \varepsilon_{2} \sigma\left(S_{2}{ }^{t} S_{2} \delta\right)\right) \\
& =\sum \exp \left(-\varepsilon_{4} \sum_{i, j} \delta_{i} s_{i j}^{2}\right) \quad\left(\varepsilon_{4}=\varepsilon^{\prime} \varepsilon_{2}\right) \\
& =\prod_{i}\left(\sum_{s \in \boldsymbol{Z}} \exp \left(-\varepsilon_{4} \delta_{i} s^{2}\right)\right)^{n-r} \leq \prod_{i}\left(1+2 \sum_{s \geq 1} \exp \left(-\varepsilon_{4} \delta_{i} s\right)\right)^{n-r} \\
& =\prod_{i}\left(1+2 \frac{\exp \left(-\varepsilon_{4} \delta_{i}\right)}{1-\exp \left(-\varepsilon_{4} \delta_{i}\right)}\right)^{n-r} \\
& =\prod_{i}\left(1+\frac{2}{\exp \left(\varepsilon_{4} \delta_{i}\right)-1}\right)^{n-r}<\prod_{i}\left(1+\frac{2}{\varepsilon_{4} \delta_{i}}\right)^{n-r} \\
& =\prod_{i}\left(\frac{\varepsilon_{4} \delta_{i}+2}{\varepsilon_{4} \delta_{i}}\right)^{n-r}<\left(\prod_{i} \varepsilon_{4}^{-1}\left(\varepsilon_{4} \varepsilon_{2}^{-1}+2\right)\right)^{n-r}|t|^{n-r} .
\end{aligned}
$$

Now the calculation before Lemma 8 implies the identity in Lemma 8 .

Q.E.D.

Lemma 9. For $t>0, t \in \Lambda_{r}^{*}$ we have 


$$
\begin{aligned}
\sum_{S_{4} \in p^{2} \Lambda_{n-r}} & \left|W_{4}+S_{4}\right|^{-k} \sqrt{\operatorname{det}\left(i^{-1}\left(W_{4}+S_{4}\right)\right)^{r}} e\left(\left(4 p^{4}\right)^{-1} \sigma\left(\left(W_{4}+S_{4}\right)^{t} S_{2} t^{-1} S_{2}\right)\right) \\
= & i^{(n-r) k}(2 \pi)^{(n-r)(k-r / 2)} 2^{(r-n)(n-r-1) / 2} p^{(r-n)(n-r+1)}\left(4 p^{4} b_{t}\right)^{(r-n)(2 k-n-1) / 2} \\
& \times \Gamma_{n-r}(k-r / 2)^{-1} e\left(\left(4 p^{4}\right)^{-1} \sigma\left(W_{4}^{t} S_{2} t^{-1} S_{2}\right)\right) \\
& \times \sum_{T}|T|^{k-(n+1) / 2} e\left(\left(4 p^{4} b_{t}\right)^{-1} \sigma\left(T W_{4}\right)\right),
\end{aligned}
$$

where $b_{t}$ is any fixed natural number such that $b_{t} t^{-1}$ is integral, $S_{2} \in M_{r, n-r}(Z)$, and $T$ runs over

$$
\left\{T \mid T>0, T \in \Lambda_{n-r}^{*},\left(4 p^{2}\right)^{-1}\left({ }^{t} S_{2} t^{-1} S_{2}+b_{t}^{-1} T\right) \in \Lambda_{n-r}^{*}\right\} .
$$

Proof. The left side equals

$$
\begin{aligned}
& i^{k(n-r)} e\left(\left(4 p^{4}\right)^{-1} \sigma\left(W_{4}{ }^{t} S_{2} t^{-1} S_{2}\right)\right) \sum_{S_{4} \in A_{n-r}}\left|-i\left(W_{4}+p^{2} S_{4}\right)\right|^{-(k-r / 2)} e\left(\left(4 p^{2}\right)^{-1} \sigma\left(S_{4}{ }^{t} S_{2} t^{-1} S_{2}\right)\right) \\
& =i^{k(n-r)} e\left(\left(4 p^{4}\right)^{-1} \sigma\left(W_{4}^{t} S_{2} t^{-1} S_{2}\right)\right) \sum_{S_{4}^{\prime} \in A_{n-r} \bmod 4 p^{2} b_{t}} e\left(\left(4 p^{2}\right)^{-1} \sigma\left(S_{4}^{\prime t} S_{2} t^{-1} S_{2}\right)\right) \\
& \times \sum_{S_{4} \in A_{n-r}}\left|-i\left(W_{4}+p^{2} S_{4}^{\prime}+4 p^{4} b_{t} S_{4}\right)\right|^{-(k-r / 2)} \\
& =i^{k(n-r)} e\left((4 p)^{-1} \sigma\left(W_{4}^{t} S_{2} t^{-1} S_{2}\right)\right) \sum_{S_{4} \in A_{n-r} \bmod 4 p^{2} b_{t}} e\left(\left(4 p^{2}\right)^{-1} \sigma\left(S_{4}^{\prime t} S_{2} t^{-1} S_{2}\right)\right) \\
& \times(2 \pi)^{(r-n)(k-r / 2)} 2^{(r-n)(n-r-1) / 2}(2 \pi)^{2(n-r)(k-r / 2)} \Gamma_{n-r}(k-r / 2)^{-1} \\
& \times\left(4 p^{4} b_{t}\right)^{-(n-r)(k-r / 2)} \sum_{\substack{T>0 \\
T \in A_{n-r}^{*}}}|T|^{k-(n+1) / 2} e\left(\left(4 p^{4} b_{t}\right)^{-1} \sigma\left(T\left(W_{4}+p^{2} S_{4}^{\prime}\right)\right) .\right.
\end{aligned}
$$

From

$$
\begin{array}{r}
\sum_{S_{4}^{\prime} \in A_{n}-r \text { mod } 4 p^{2} b_{t}} e\left(\left(4 p^{2}\right)^{-1} \sigma\left(S_{4}^{\prime t} S_{2} t^{-1} S_{2}\right)+\left(4 p^{4} b_{t}\right)^{-1} \sigma\left(p^{2} T S_{4}^{\prime}\right)\right) \\
=\left\{\begin{array}{lc}
\left(4 p^{2} b_{t}\right)^{(n-r)(n-r+1) / 2} & \text { if }\left(4 p^{2}\right)^{-1}\left({ }^{t} S_{2} t^{-1} S_{2}+b_{t}^{-1} T\right) \in \Lambda_{n-r}^{*}, \\
0 & \text { otherwise }
\end{array}\right.
\end{array}
$$

follows the identity in Lemma 9.

Q.E.D.

Now we have

$$
\begin{aligned}
\sum_{S=\left(\begin{array}{c}
0 \\
t S_{2} \\
S_{4}
\end{array}\right) \in p_{2}^{2} A_{n}}\left(f \mid N\left(\frac{1}{0} \mid \frac{C^{-1} S^{t} C^{-1}}{1}\right)\right)(Z) & \sum_{S_{4} \in p^{2} A_{n-r}}|C|^{k} 2^{r(r-n) / 2} p^{2 r(r-n)} \sum_{\substack{t>0 \\
t \in \Lambda_{r}^{*} \\
S_{2} \in M_{r, n-r}(Z)}}|t|^{(r-n) / 2} b(t) \\
& \times e\left(\sigma\left(t W_{1}\right)-p^{-2} \sigma\left(W_{2}^{t} S_{2}\right)\right)\left|W_{4}+S_{4}\right|^{-k} \sqrt{\operatorname{det}\left(i^{-1}\left(W_{4}+S_{4}\right)\right)^{r}} \\
& \times e\left(\left(4 p^{4}\right)^{-1} \sigma\left(\left(W_{4}+S_{4}\right)^{t} S_{2} t^{-1} S_{2}\right)\right) \\
= & |C|^{k} 2^{(r-n)(n-1) / 2} p^{(r-n)(n+r+1)} i^{(n-r) k}(2 \pi)^{(n-r)(k-r / 2)} \\
& \times \Gamma_{n-r}(k-r / 2)^{-1} \sum_{t, S_{2}, T}|t|^{(r-n) / 2}\left(4 p^{4} b_{t}\right)^{(r-n)(2 k-n-1) / 2}
\end{aligned}
$$




$$
\begin{aligned}
\times|T|^{k-(n+1) / 2} b(t) e\left(\sigma\left(t W_{1}\right)-p^{-2} \sigma\left(W_{2}{ }^{t} S_{2}\right)+\left(4 p^{4}\right)^{-1} \sigma\left(W_{4}{ }^{t} S_{2} t^{-1} S_{2}\right)\right. \\
\left.+\left(4 p^{4} b_{t}\right)^{-1} \sigma\left(T W_{4}\right)\right),
\end{aligned}
$$

where

$$
\begin{aligned}
& t>0, \quad t \in \Lambda_{r}^{*}, \quad S_{2} \in M_{r, n-r}(Z), \quad T>0, \\
& T \in \Lambda_{n-r}^{*}, \quad\left(4 p^{2}\right)^{-1}\left({ }^{t} S_{2} t^{-1} S_{2}+b_{t}^{-1} T\right) \in \Lambda_{n-r}^{*} .
\end{aligned}
$$

Put

$$
P=\left(\begin{array}{cc}
t & -\left(2 p^{2}\right)^{-1} S_{2} \\
-\left(2 p^{2}\right)^{-1}{ }^{t} S_{2} & \left(4 p^{4}\right)^{-1}\left(b_{t}^{-1} T+{ }^{t} S_{2} t^{-1} S_{2}\right)
\end{array}\right)=\left(\begin{array}{cc}
P_{1} & P_{2} \\
{ }^{t} P_{2} & P_{4}
\end{array}\right)
$$

then

$$
P=\left(\begin{array}{cc}
1 & 0 \\
-\left(2 p^{2}\right)^{-1}{ }^{t} S_{2} t^{-1} & 1
\end{array}\right)\left(\begin{array}{cc}
t & 0 \\
0 & \left(4 p^{4} b_{t}\right)^{-1} T
\end{array}\right)\left(\begin{array}{cc}
1 & -\left(2 p^{2}\right)^{-1} t^{-1} S_{2} \\
0 & 1
\end{array}\right)
$$

implies that $P$ is positive definite and $|P|=\left(4 p^{4} b_{t}\right)^{r-n}|t||T|$. Our assumptions on $t, S_{2}, T$ mean that $P_{1} \in \Lambda_{r}^{*}, 2 p^{2} P_{2} \in M_{r, n-r}(Z), p^{2} P_{4} \in \Lambda_{n-r}^{*}$, and $b_{t} 4 p^{4} P_{4}$ $-b_{t}{ }^{t} S_{2} t^{-1} S_{2} \in \Lambda_{n-r}^{*} . \quad\left\{t, S_{2}, T\right\}$ and $P$ correspond bijectively and

$$
\sigma(W P)=\sigma\left(W_{1} t\right)-p^{-2} \sigma\left(W_{2}^{t} S_{2}\right)+\left(4 p^{4}\right)^{-1} \sigma\left(W_{4}\left(b_{t}^{-1} T+{ }^{t} S_{2} t^{-1} S_{2}\right)\right) .
$$

Thus we have

LEMma 10.

$$
\begin{aligned}
\sum_{S=\left(t_{t_{2}}^{0} \sum_{S_{4}}^{S_{2}}\right) \in p^{2} A_{n}}\left(f \mid N\left(\frac{1}{0} \mid \frac{C^{-1} S^{t} C^{-1}}{1}\right)\right)(Z) \\
=|C|^{k} 2^{(r-n)(n-1) / 2} p^{(r-n)(n+r+1)} i^{(n-r) k}(2 \pi)^{(n-r)(k-r / 2)} \cdot \\
\quad \times \Gamma_{n-r}(k-r / 2)^{-1} \sum_{P>0} b\left(P_{1}\right)\left|P_{1}\right|^{(r+1) / 2-k}|P|^{k-(n+1) / 2} e(\sigma(P W)),
\end{aligned}
$$

where

$$
P_{1} \in \Lambda_{r}^{*}, \quad 2 p^{2} P_{2} \in M_{r, n-r}(Z), \quad p^{2} P_{4} \in \Lambda_{n-r}^{*} .
$$

Lemma 11. Put $G=\left\{\left({ }^{t} S_{2}{ }^{t} C_{1}^{-1}-S_{4}{ }^{t}\left(C_{1}^{-1} C_{2} C_{4}^{-1}\right), S_{4}{ }^{t} C_{4}^{-1}\right) \mid S_{2} \in p^{2} M_{r, n-r}(Z)\right.$, $\left.S_{4} \in p^{2} \Lambda_{n-r}\right\}, \quad G^{\prime}=\left\{\left(C_{4}{ }^{t} S_{2}, C_{4} S_{4}\right) \mid S_{2} \in M_{r, n-r}(Z), S_{4} \in \Lambda_{n-r}\right\}$. Then we have $\left[G^{\prime}: G\right]=p^{(n-r)(n+r+1)} a b s\left(\left|C_{1}\right|^{r-n}\left|C_{4}\right|^{-n-1}\right)$.

Proof. By definition $C=\left(\begin{array}{ll}C_{1} & C_{2} \\ & C_{4}\end{array}\right)$ and $p C^{-1}=p\left(\begin{array}{c}C_{1}^{-1}-C_{1}^{-1} C_{2} C_{4}^{-1} \\ C_{4}^{-1}\end{array}\right)$ are integral. It implies $G^{\prime} \supset G$. Put $G_{0}=M_{n-r, r}(Z) \times\left\{C_{4} S_{4} \mid S_{4} \in \Lambda_{n-r}\right\}$; then $\left[G_{0}: G^{\prime}\right]=a b s\left|C_{4}\right|^{r}$. As representatives of $G_{0} / G$ we can take representatives of $\left\{C_{4} S_{4} \mid S_{4} \in \Lambda_{n-r}\right\} /\left\{S_{4}{ }^{t} C_{4}^{-1} \mid S_{4} \in p^{2} \Lambda_{n-r}\right\}$ and then representatives of 


$$
M_{n-r, r}(Z) /\left\{{ }^{t} S_{2}{ }^{t} C_{1}^{-1} \mid S_{2} \in p^{2} M_{r, r-n}(Z)\right\}
$$

Hence we have

$$
\begin{aligned}
{\left[G_{0}: G\right]=} & {\left[\left\{C_{4} S_{4} \mid S_{4} \in \Lambda_{n-r}\right\}:\left\{S_{4}{ }^{t} C_{4}^{-1} \mid S_{4} \in p^{2} \Lambda_{n-r}\right\}\right] } \\
& \times\left[M_{n-r, r}(Z):\left\{{ }^{t} S_{2}{ }^{t} C_{1}^{-1} \mid S_{2} \in p^{2} M_{n-r, r}(Z)\right\}\right] \\
= & {\left[\Lambda_{n-r}: p^{2} C_{4}^{-1} \Lambda_{n-r}{ }^{t} C_{4}^{-1}\right] a b s\left|p^{2}{ }^{t} C_{1}^{-1}\right|^{n-r} } \\
= & a b s\left|p C_{4}^{-1}\right|^{n-r+1}\left|p^{2}{ }^{t} C_{1}^{-1}\right|^{n-r} .
\end{aligned}
$$

Thus we have $\left[G^{\prime}: G\right]=p^{(n-r)(n+r+1)} a b s\left(\left|C_{1}\right|^{r-n}\left|C_{4}\right|^{-n-1}\right)$.

Q.E.D.

It is obvious that

$$
\begin{aligned}
G & =\left\{\widetilde{S^{t} C^{-1}} \mid S=\left(\begin{array}{cc}
0 & S_{2} \\
{ }^{t} S_{2} & S_{4}
\end{array}\right) \in p^{2} \Lambda_{n}\right\}, \\
G^{\prime} & =\left\{\widetilde{C S} \mid S=\left(\begin{array}{cc}
0 & S_{2} \\
{ }^{t} S_{2} & S_{4}
\end{array}\right) \in \Lambda_{n}\right\}
\end{aligned}
$$

and

$$
\widetilde{N\left(\frac{1}{0} \mid \frac{C^{-1} S^{t} C^{-1}}{1}\right)}=\left(\tilde{C}, \tilde{D}+\widetilde{S^{t} C^{-1}}\right), \quad \widetilde{N\left(\frac{1}{0} \mid \frac{S}{1}\right)}=(\tilde{C}, \tilde{D}+\widetilde{C S}) .
$$

We take

$$
S_{i}^{\prime}=\left(\begin{array}{cc}
0 & S_{i, 2} \\
{ }^{t} S_{i, 2} & S_{i, 4}
\end{array}\right) \in \Lambda_{n}
$$

such that $\widetilde{C S_{i}^{\prime}}$ is representatives of $G^{\prime} / G$. For $M \in \Gamma_{n},(f \mid M)(Z)$ is uniquely determined by $\tilde{M}$. Hence we may write $(f \mid \tilde{M})(Z)$ for $(f \mid M)(Z)$. Then we have

$$
\begin{aligned}
& \sum_{S=\left(\begin{array}{c}
0 \\
t S_{2} \\
\substack{S_{4} \\
S_{4}}
\end{array}\right) \in A_{n}}\left(f \mid N\left(\frac{1}{0} \mid \frac{S}{1}\right)\right)(Z)=\sum_{g \in G^{\prime}}(f \mid(\tilde{C}, \tilde{D}+g))(Z) \\
& =\sum_{i} \sum_{g \in G}\left(f \mid\left(\tilde{C}, \widetilde{\left(D+C S_{i}^{\prime}\right.}\right)+g\right)(Z) \\
& =\sum_{i} \sum_{S=\left(\begin{array}{c}
0 \\
t S_{2} \\
S_{4}
\end{array}\right) \in p^{2} A_{n}}\left(f \mid N\left(\frac{1}{0} \mid \frac{S_{i}^{\prime}}{1}\right)\left(\frac{1}{0} \mid \frac{C^{-1} S^{t} C^{-1}}{1}\right)\right)(Z) .
\end{aligned}
$$

For

$$
M=N\left(\frac{1}{0} \mid \frac{S_{i}^{\prime}}{1}\right)
$$

we have $C_{M}=C_{N}=C, D_{M}=C S_{i}^{\prime}+D,\left(A_{M} C_{M}^{-1}\right)_{1} \equiv 0 \bmod 1$ and $C_{M} Z^{t} C_{M}$ 
$+D_{M}{ }^{t} C_{M}=W+C S_{i}^{\prime}{ }^{t} C$. Applying Lemma 10, we have

$$
\begin{aligned}
\sum_{S=\left(c_{t}^{0} S_{S_{2}}^{S_{S_{4}}}\right) \in A_{n}}\left(f \mid N\left(\frac{1}{0} \mid \frac{S}{1}\right)\right)(Z) & \alpha|C|^{k} p^{(r-n)(n+r+1)} \sum_{P>0} b\left(P_{1}\right)\left|P_{1}\right|^{(r+1) / 2-k}|P|^{k-(n+1) / 2} \\
= & \times e\left(\sigma\left(P\left(W+C S_{i}^{\prime}{ }^{i} t C\right)\right),\right.
\end{aligned}
$$

where $\alpha=i^{(n-r) k} 2^{(r-n)(n-1) / 2}(2 \pi)^{(n-r)(k-r / 2)} \Gamma_{n-r}(k-r / 2)^{-1}$, and $P$ runs over

$$
\left\{P>0 \mid P_{1} \in \Lambda_{r}^{*}, 2 p^{2} P_{2} \in M_{r, n-r}(Z), p^{2} P_{4} \in \Lambda_{n-r}^{*}\right\} \text {. }
$$

We fix $P$ such that $P_{1} \in \Lambda_{r}^{*}, 2 p^{2} P_{2} \in M_{r, n-r}(Z), p^{2} P_{4} \in \Lambda_{n-r}^{*}$ and we put $\chi(g)=e\left(2 \sigma\left(g_{1}{ }^{t} C_{1} P_{2}\right)+2 \sigma\left(g_{2}{ }^{t} C_{2} P_{2}\right)+\sigma\left(g_{2}{ }^{t} C_{4} P_{4}\right)\right)$ for $g=\left(g_{1}^{(n-r, r)}, g_{2}^{(n-r, n-r)}\right) \in G^{\prime}$. It is easy to see that $\chi(g)=1$ for $g \in G$ and $e\left(\sigma\left(P C S^{t} C\right)\right)=\chi\left(\left(C_{4}{ }^{t} S_{2}, C_{4} S_{4}\right)\right)$ $=\chi(\widetilde{C S})$ for $S=\left(\begin{array}{cc}0 & S_{2} \\ { }^{t} S_{2} & S_{4}\end{array}\right) \in \Lambda_{n}$. Therefore the definition of $S_{i}^{\prime}$ implies

$$
\sum_{i} e\left(\sigma\left(P C S_{i}^{\prime t} C\right)\right)=\sum_{g \in G^{\prime} / G} \chi(g) .
$$

$\chi$ is trivial if and only if $2^{t} C_{1} P_{2} C_{4} \in M_{r, n-r}(Z)$ and ${ }^{t} C_{2} P_{2} C_{4}+{ }^{t} C_{4}{ }^{t} P_{2} C_{2}+$ ${ }^{t} C_{4} P_{4} C_{4} \in \Lambda_{n-r}^{*}$. Put $T={ }^{t} C P C$; then we have

Lemma 12. $P_{1} \in \Lambda_{r}^{*}, 2 p^{2} P_{2} \in M_{r, n-r}(Z), p^{2} P_{4} \in \Lambda_{n-r}^{*}, 2^{t} C_{1} P_{2} C_{4} \in M_{r, n-r}(Z)$ and ${ }^{t} C_{2} P_{2} C_{4}+{ }^{t} C_{4}^{t} P_{2} C_{2}+{ }^{t} C_{4} P_{4} C_{4} \in \Lambda_{n-r}^{*}$ if and only if $T \in \Lambda_{n}^{*}$ and ${ }^{t} C_{1}^{-1} T_{1} C_{1}^{-1}$ $=\left({ }^{t} C^{-1} T C^{-1}\right)_{1} \in \Lambda_{r}^{*}$.

Proof. $T={ }^{t} C P C$ implies $T_{1}={ }^{t} C_{1} P_{1} C_{1}, T_{2}={ }^{t} C_{1} P_{1} C_{2}+{ }^{t} C_{1} P_{2} C_{4}, T_{4}=$ ${ }^{t} C_{2} P_{1} C_{2}+{ }^{t} C_{4}{ }^{t} P_{2} C_{2}+{ }^{t} C_{2} P_{2} C_{4}+{ }^{t} C_{4} P_{4} C_{4}$. The assumptions on $P$ imply ${ }^{t} C_{1}^{-1} T_{1} C_{1}^{-1}=P_{1} \in \Lambda_{r}^{*}$ and so $T_{1} \in \Lambda_{r}^{*} . \quad 2 T_{2}=2^{t} C_{1} P_{1} C_{2}+2^{t} C_{1} P_{2} C_{4} \in M_{r, n-r}(Z)$ holds since $P_{1} \in \Lambda_{r}^{*}, 2^{t} C_{1} P_{2} C_{4} \in M_{r, n-r}(Z) . \quad T_{4}={ }^{t} C_{2} P_{1} C_{2}+\left({ }^{t} C_{4}{ }^{t} P_{2} C_{2}+{ }^{t} C_{2} P_{2} C_{4}\right.$ $\left.+{ }^{t} C_{4} P_{4} C_{4}\right) \in \Lambda_{n-r}^{*}$. Thus "only if" part has been proved. Conversely, assume $T \in \Lambda_{n}^{*},{ }^{t} C_{1}^{-1} T_{1} C_{1}^{-1} \in \Lambda_{r}^{*}$. Then $P_{1}={ }^{t} C_{1}^{-1} T_{1} C_{1}^{-1} \in \Lambda_{r}^{*},{ }^{t} C_{2} P_{2} C_{4}+{ }^{t} C_{4}{ }^{t} P_{2} C_{2}$ $+{ }^{t} C_{4} P_{4} C_{4}=T_{4}-{ }^{t} C_{2} P_{1} C_{2} \in \Lambda_{n-r}^{*}, 2^{t} C_{1} P_{2} C_{4}=2 T_{2}-2 C_{1} P_{1} C_{2} \in M_{r, n-r}(Z), 2 p^{2} P_{2}$ $=2\left(p^{t} C_{1}^{-1}\right)\left({ }^{t} C_{1} P_{2} C_{4}\right)\left(p C_{4}^{-1}\right) \in M_{r, n-r}(Z)$ follow easily. From $P_{4}-{ }^{t} C_{4}^{-1} T_{4} C_{4}^{-1}+$ ${ }^{t} C_{4}^{-1 t}{ }^{t} C_{2} P_{1} C_{2} C_{4}^{-1}=-{ }^{t} P_{2} C_{2} C_{4}^{-1}-{ }^{t} C_{4}^{-1}{ }^{t} C_{2} P_{2}=-{ }^{t} C_{4}^{-1}\left({ }^{t} T_{2}-{ }^{t} C_{2} P_{1} C_{1}\right) C_{1}^{-1} C_{2} C_{4}^{-1}-$ ${ }^{t}\left(C_{1}^{-1} C_{2} C_{4}^{-1}\right)\left(T_{2}-{ }^{t} C_{1} P_{1} C_{2}\right) C_{4}^{-1}$ and $p C^{-1}=p\left(\begin{array}{cc}C_{1}^{-1}-C_{1}^{-1} C_{2} C_{4}^{-1} \\ 0 & C_{4}^{-1}\end{array}\right) \in M_{n}(Z)$ follows $p^{2} P_{4} \in \Lambda_{n-r}^{*}$.

Q.E.D.

Summarizing we have

LEMma 13. 


$$
\begin{gathered}
\sum_{S=\left(\begin{array}{c}
\left.t_{S_{2}}^{0} S_{S_{2}}^{S_{2}}\right) \in A_{n} \\
=
\end{array}\right.}\left(f \mid N\left(\frac{1}{0} \mid \frac{S}{1}\right)\right)(Z) \\
=\left.\alpha C_{1}\right|^{k}\left|C_{4}\right|^{-k} \sum_{\substack{T>0 \\
T \in \Lambda_{n}^{*}}} b\left(\left({ }^{t} C^{-1} T C^{-1}\right)_{1}\right)\left|T_{1}\right|^{(r+1) / 2-k} \\
\times|T|^{k-(n+1) / 2} e\left(\sigma\left(T C^{-1} D\right)\right) e(\sigma(T Z))
\end{gathered}
$$

where we suppose $b\left(\left({ }^{t} C^{-1} T C^{-1}\right)_{1}\right)=0$ if $\left({ }^{t} C^{-1} T C^{-1}\right)_{1} \notin \Lambda_{r}^{*}$.

This lemma, Proposition 1 and Theorem in $\S 1$ imply the former part of the following

TheOREm. Let $r, n, k$, be natural numbers such that $1 \leq r \leq n-1$, $k \geq n+r+2, k \equiv 0 \bmod 2$, and let $f\left(z^{(r)}\right)=\sum_{t>0} b(t) e(\sigma(t z))$ be a cusp form of degree $r$ and weight $k$. If we put

$$
E_{n, r}^{k}(Z, f)=\sum_{M \in \Delta_{n, r} \backslash \Gamma_{n}}(f \mid M)(Z)=\sum_{\substack{T \geq 0 \\ T \in \Lambda^{*}}} a(T, f) e(\sigma(T Z)),
$$

then we have

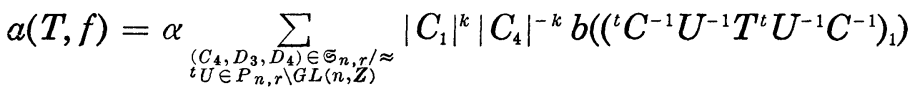

$$
\begin{aligned}
& \times\left|\left(U^{-1} T^{t} U^{-1}\right)_{1}\right|^{(r+1) / 2-k}|T|^{k-(n+1) / 2} e\left(\sigma\left(U^{-1} T^{t} U^{-1} C^{-1} D\right)\right)
\end{aligned}
$$

for $T>0$, where $M_{1}$ stands for the upper left $r \times r$ matrix of $M,(C, D)$ stands for any fixed symmetric coprime pair such that

$$
(\tilde{C}, \tilde{D})=\left(0, C_{4}, D_{3}, D_{4}\right), \quad C=\left(\begin{array}{cc}
C_{1} & C_{2} \\
0 & C_{4}
\end{array}\right), \quad|C| \neq 0
$$

and $\left(A C^{-1}\right)_{1} \equiv 0 \bmod 1$ for some $\left(\begin{array}{ll}A & B \\ C & D\end{array}\right) \in \Gamma_{n}$, and

$$
\begin{aligned}
\alpha= & i^{(n-r) k} 2^{(r-n)(n-1) / 2}(2 \pi)^{(n-r)(k-r / 2)} \\
& \times\left(\pi^{(n-r)(n-r-1) / 4} \prod_{\nu=0}^{n-r-1} \Gamma(k-(r+\nu) / 2)\right)^{-1} .
\end{aligned}
$$

Moreover we have $a(T, f)=O\left(\left|T_{1}\right|^{-(k-r-1) / 2}|T|^{k-(n+1) / 2}\right)$ if $T>0$ runs over any fixed Siegel domain.

To prove the latter part we prepare the following

Lemma 14. The number of $\left(D_{3}, D_{4}\right)$ such that $\left(C_{4}, D_{3}, D_{4}\right)$ is representatives of $\mathfrak{S}_{n, r} \mid \approx$ for fixed $C_{4}\left(\left|C_{4}\right| \neq 0\right)$ is at most abs $\left|C_{4}\right|^{r} \delta_{1}^{n-r} \cdots \delta_{n-r}$ where $\delta_{1}|\cdots| \delta_{n-r}$ are elementary divisors of $C_{4}$. 
Proof. By the definition of the relation $\approx$ the number of inequivalent $\left(C_{4}, D_{3}, D_{4}\right)$ for fixed $C_{4}$ is at most $\left[M_{n-r, r}(Z): C_{4} M_{n-r, r}(Z)\right] \times$ $\left[\left\{D_{4} \in M_{n-r}(Z) \mid C_{4}^{-1} D_{4}={ }^{t}\left(C_{4}^{-1} D_{4}\right)\right\}: C_{4} \Lambda_{n-r}\right] . \quad\left[M_{n-r, r}(Z): C_{4} M_{n-r, r}(Z)\right]=a b s\left|C_{4}\right|^{r}$ is obvious. The latter part equals $\left[\left\{S={ }^{t} S \in M_{n-r}(Q) \mid C_{4} S \in M_{n-r}(Z)\right\}: \Lambda_{n-r}\right]$. Put $C_{4}=g_{1} \delta g_{2}$ where $g_{i} \in G L(n-r, Z)$,

$$
\delta=\left(\begin{array}{ccc}
\delta_{1} & & \\
& \ddots & \\
& & \delta_{n-r}
\end{array}\right), \quad \delta_{1}|\cdots| \delta_{n-r} .
$$

Then $\left\{S={ }^{t} S \in M_{n-r}(\boldsymbol{Q}) \mid C_{4} S \in M_{n-r}(Z)\right\}=\left\{S={ }^{t} S \in M_{n-r}(\boldsymbol{Q}) \mid \delta S \in M_{m-r}(Z)\right\}$. Hence the latter part equals $\delta_{1}^{n-r} \cdots \delta_{n-r}$.

Q.E.D.

We can take

$$
\left\{\left(\begin{array}{ccc}
c_{1} & & \\
& \ddots & c_{i j} \\
0 & & \\
& & c_{n-r}
\end{array}\right) \mid c_{1}, \cdots, c_{n-r}>0,0 \leq c_{i \jmath}<c_{\vartheta}\right\}
$$

as representatives of $G L(n-r, Z) \backslash\left\{C_{4} \in M_{n-r}(Z)|| C_{4} \mid \neq 0\right\}$. It

$$
C_{4}=\left(\begin{array}{ccc}
c_{1} & & \\
& \ddots & c_{i j} \\
& & c_{n-r}
\end{array}\right) \text {, }
$$

then $\delta_{1}^{n-r} \cdots \delta_{n-r}=\delta_{1}\left(\delta_{1} \delta_{2}\right) \cdots\left(\delta_{1} \cdots \delta_{n-r}\right) \leq c_{1}\left(c_{1} c_{2}\right) \cdots\left(c_{1} \cdots c_{n-r}\right)$ where $\delta_{1}|\cdots| \delta_{n-r}$ are elementary divisors of $C_{4}$. Hence we have

$$
\begin{aligned}
& a(T, f)=O\left(\sum_{\substack{\left(C_{4}, D_{3}, D_{4}\right) \in \in_{n, r} \approx \\
t U \in P(n, r) \in L(n, \mathbb{Z})}}\left|C_{4}\right|^{-k} \times\left|\left(U^{-1} T^{t} U^{-1}\right)_{1}\right|^{(r+1-k) / 2}|T|^{k-(n+1) / 2}\right) \\
& =\sum_{c_{i}=1}^{\infty}\left(\prod_{i=1}^{n-r} c_{i}\right)^{-k+r}\left(c_{1}^{n-r} \cdots c_{n-r}\right)\left(c_{2} \cdots c_{n-r}^{n-r-1}\right) \\
& \times O\left(\sum_{t_{U \in P}} \sum_{n, r \backslash G L(n, Z)}\left|\left(U^{-1} T^{t} U^{-1}\right)_{1}\right|^{(r+1-k) / 2}|T|^{k-(n+1) / 2}\right),
\end{aligned}
$$

where the sum of $c_{i}$ is equal to $\zeta(k-n)^{n-r}$, and the last sum is a socalled Selberg's zeta function and the order of the magnitude is $\left|T_{1}\right|^{(r+1-k) / 2}|T|^{k-(n+1) / 2}$ if $T$ runs over any fixed Siegel domain (p. 143 and Theorem in p. 144 in [5]). This completes the proof of Theorem.

\$3.

Let $k, n$ be natural numbers such that $k \geq n+2, k \equiv 0 \bmod 2$ and put 


$$
E_{n}^{k}(Z)=\sum|C Z+D|^{-k} \quad\left(Z \in H_{n}\right),
$$

where $(C, D)$ runs over representatives of $G L(n, Z) \backslash\{$ symmetric coprime pairs\}. Our aim is to prove

Theorem. Put

$$
E_{n}^{k}(Z)=\sum_{\substack{T \geq 0 \\ T \in \Lambda_{n}^{*}}} a(T) e(\sigma(T Z))
$$

Then there are positive numbers $c_{1}, c_{2}$ such that

$$
c_{1}|T|^{k-(n+1) / 2}<|a(T)|<c_{2}|T|^{k-(n+1) / 2} \quad \text { for } T>0 \text {. }
$$

Put $S_{T}=\sum_{R} e(-\sigma(T R)) \nu(R)^{-k}$ for $T \in \Lambda_{n}^{*}$ where $R$ runs over all $n \times n$ rational symmetric matrices modulo 1 and $\nu(R)$ is the product of denominators of elementary divisors of $R$. Then it is known that

$$
a(T)=\text { const. } \times|T|^{k-(n+1) / 2} S_{T} \quad \text { for } T>0 \text { ([9]) . }
$$

On the other hand $S_{T}=\sum|C|^{-k} e\left(-\sigma\left(T C^{-1} D\right)\right)$ where $(C, D)$ runs over the set $\{(C, D) \mid$ symmetric coprime pair, $|C| \neq 0\} / \sim$. Here by definition $\left(C^{\prime}, D^{\prime}\right) \sim(C, D)$ if and only if there are $U \in G L(n, Z), S \in A_{n}$ such that $\left(C^{\prime}, D^{\prime}\right)=U(C, D+C S)$. We take as representatives of $C$ matrices of the form

$$
\left(\begin{array}{ccc}
c_{1} & & \\
& \ddots & c_{i j} \\
& & c_{n}
\end{array}\right), \quad 0 \leq c_{i j}<c_{j}
$$

then the number of the choice of $D$ for $C$ is at most $c_{1}^{n} \cdots c_{n}$ as in the proof of Theorem in $\S 2$. Thus we have

$$
\left|S_{T}\right| \leq \sum_{c_{i}=1}^{\infty}\left(\prod c_{i}\right)^{-k}\left(\prod c_{i}^{i-1}\right) \prod c_{i}^{n+1-i}=\zeta(k-n)^{n} .
$$

To complete the proof we must show $\left|S_{T}\right|>\varepsilon$ where $\varepsilon$ is a positive number independent of $T$.

Put $S_{p}(T)=\sum_{R} e(-\sigma(T R)) \nu(R)^{-k}$ where $R$ runs over all $n \times n$ rational symmetric matrices modulo 1 such that $\nu(R)$ is a power of $p$. Then $S_{T}=\prod_{p} S_{p}(T)$ for $T>0, T \in \Lambda_{n}^{*}$. Put

$$
J=\frac{1}{2}\left(\begin{array}{rr}
0 & 1_{k} \\
1_{k} & 0
\end{array}\right) \text { and } A_{q}(T)=\#\left\{C \in M_{2 k, n}(Z) \bmod q ! q^{-1}\left({ }^{t} C J C-T\right) \in \Lambda_{n}^{*}\right\} .
$$


Then we have

$$
S_{p}(T)=\left(p^{a}\right)^{n(n+1) / 2-2 k n} A_{p a}(T)
$$

for sufficiently large $a$.

Lemma 1. For $W \in \Lambda_{n}$ we put

$$
G\left(W ; p^{a}\right)=\sum_{\substack{C \in M_{2 k, n}(Z) \\ C \text { mod } p^{a} \\ \text { primitive mod } p}} e\left(p^{-a} \sigma\left({ }^{t} C J C W\right)\right) .
$$

Then we have

$$
G\left(W ; p^{a}\right)=\left\{\begin{array}{cl}
p^{2 k n(a-1)} G\left(p^{-(a-1)} W ; p\right) & \text { if } W \equiv 0 \bmod p^{a-1} \\
0 & \text { otherwise. }
\end{array}\right.
$$

Proof. For a primitive element $C_{1}$ of $M_{2 k, n}(Z)$ we take a unimodular matrix $U=\left(C_{1} *\right)$. From

$$
\left(\begin{array}{l}
W \\
0
\end{array}\right)=\left(\begin{array}{l}
1_{n} \\
0
\end{array}\right) W=U^{-1} C_{1} W
$$

follows that $W \equiv 0 \bmod p$ if and only if $C_{1} W \equiv 0 \bmod p$. Lemma is obvious for $a=1$. We assume $a \geq 2$. Decompose $C \in M_{2 k, n}(Z)$ as $C=C_{1}$ $+p^{a-1} C_{2}$. Then $C$ is primitive $\bmod p$ if and only if $C_{1}$ is primitive $\bmod p$.

Hence we have

$$
G\left(W ; p^{a}\right)=\sum_{\substack{C_{1} \text { mod } \\ C_{1}: \text { primitive } \bmod p}} e\left(p^{-a} \sigma\left({ }^{t} C_{1} J C_{1} W\right)\right) \sum_{C_{2} \bmod p} e\left(2 p^{-1} \sigma\left({ }^{t} C_{1} J C_{2} W\right)\right) .
$$

$2 \sigma\left({ }^{t} C_{1} J C_{2} W\right) \equiv 0 \bmod p$ for any $C_{2}$ if and only if $2 W^{t} C_{1} J \equiv 0 \bmod p$, and it is equivalent to $W \equiv 0 \bmod p$. Thus we have

$$
G\left(W ; p^{a}\right)=\left\{\begin{array}{cl}
p^{2 k n} G\left(p^{-1} W ; p^{a-1}\right) & \text { if } W \equiv 0 \bmod p, \\
0 & \text { otherwise. }
\end{array}\right.
$$

Now our lemma is inductively proved.

Q.E.D.

Put

$$
A_{p^{a}}^{\prime}(T)=\#\left\{C \in M_{2 k, n}(Z) \bmod p^{a} \mid \begin{array}{l}
C ; \text { primitive } \bmod p, \\
p^{-a}\left({ }^{t} C J C-T\right) \in \Lambda_{n}^{*}
\end{array}\right\} .
$$

LEMma 2.

$$
\left(p^{a}\right)^{n(n+1) / 2-2 k n} A_{p^{a}}^{\prime}(T)=p^{n(n+1) / 2-2 k n} A_{p}^{\prime}(T) \quad \text { for } a \geq 1, T \in \Lambda_{n}^{*} .
$$


Proof.

$$
\begin{aligned}
\left(p^{a}\right)^{n(n+1) / 2} A_{p^{a}}^{\prime}(T) & =\sum_{W \in \Lambda_{n} \bmod p^{a}} \sum_{\substack{C \text { mod } p^{a} \\
\text { primitive mod } p}} e\left(p^{-a} \sigma\left(\left({ }^{t} C J C-T\right) W\right)\right) \\
& =\sum_{W \in \Lambda_{n} \bmod p^{a}} G\left(W ; p^{a}\right) e\left(-p^{-a} \sigma(T W)\right) \\
& =\sum_{W \in \Lambda_{n} \bmod p} p^{2 k n(a-1)} G(W ; p) e\left(-p^{-1} \sigma(T W)\right) \\
& =p^{2 k n(a-1)} p^{n(n+1) / 2} A_{p}^{\prime}(T) .
\end{aligned}
$$

LEMma 3.

$$
S_{p}(T) \geq p^{n(n+1) / 2-2 k n} A_{p}^{\prime}(T) \quad \text { for } T \in \Lambda_{n}^{*} .
$$

Proof. This follows from $A_{p a}(T) \geq A_{p^{a}}^{\prime}(T)$. Q.E.D.

Lemma 4. There is a positive number $\varepsilon$ such that $S_{2}(T)>\varepsilon$ for $T \in \Lambda_{n}^{*}$.

Proof. $A_{2}^{\prime}(T)$ is uniquely determined by $T \bmod 2$. Hence the values of $A_{2}^{\prime}(T)$ is a finite set. Hence we have only to prove $A_{2}^{\prime}(T) \neq 0$ for $T \in \Lambda_{n}^{*}$. By the theory of quadratic forms $T \in \Lambda_{n}^{*}$ is equivalent over $Z_{2}$ to a direct sum of

$$
2^{a-1}\left(\begin{array}{ll}
0 & 1 \\
1 & 0
\end{array}\right), \quad 2^{a-1}\left(\begin{array}{ll}
2 & 1 \\
1 & 2
\end{array}\right), \quad 2^{a} u\left(a \geq 0, u \in Z_{2}^{\times}\right) .
$$

Since $A_{2}^{\prime}\left(T+2 T^{\prime}\right)=A_{2}^{\prime}(T)$ for $T, T^{\prime} \in \Lambda_{n}^{*}$, we may assume that $T$ is a direct sum of

$$
\frac{1}{2}\left(\begin{array}{ll}
0 & 1 \\
1 & 0
\end{array}\right), \quad \frac{1}{2}\left(\begin{array}{ll}
2 & 1 \\
1 & 2
\end{array}\right), \quad u \in Z_{2}^{\times}
$$

and 0 . Hence we may suppose

$$
T=\left\{\begin{array}{llllll}
A_{1} & & & & & \\
& \ddots & & & & \\
& & & & & \\
& & A_{r} & & \\
& & u_{1} & & \\
& & & \ddots & \\
& & & & \ddots & \\
& & & & & u_{n-2 r}
\end{array}\right\}
$$

where

$$
A_{1}=\cdots=A_{r-1}=\frac{1}{2}\left(\begin{array}{ll}
0 & 1 \\
1 & 0
\end{array}\right), \quad A_{r}=\frac{1}{2}\left(\begin{array}{ll}
0 & 1 \\
1 & 0
\end{array}\right) \quad \text { or } \quad \frac{1}{2}\left(\begin{array}{ll}
2 & 1 \\
1 & 2
\end{array}\right)
$$


$u_{3}=\cdots=u_{n-2 r}=0$ and $u_{1}, u_{2}=0$ or $\in Z_{2}^{\times}$(93:18 in [6]). Denote by $t$ the number of $u_{i}$ such that $u_{i}=0$. It is easy to see that $\frac{1}{r+t-1} \frac{1}{2}\left(\begin{array}{ll}0 & 1 \\ 1 & 0\end{array}\right)$ represents primitively ${\underset{i}{i=1}}_{i}^{r-1} A_{i} \frac{1}{t}(0)$. To prove $A_{2}^{\prime}(T) \neq 0$ we have only to show that $\underset{k-(r+t-1)}{\frac{1}{2}}\left(\begin{array}{ll}0 & 1 \\ 1 & 0\end{array}\right)$ represents $A_{r}, A_{r} \perp\left(u_{1}\right), A_{r} \perp\left(u_{1}\right) \perp\left(u_{2}\right),\left(u_{i} \epsilon\right.$ $\left.Z_{2}^{\times}\right)$, primitively according as $t=n-2 r, n-2 r-1, n-2 r-2$.

(i) The case of $t=n-2 r, n-2 r-1$; then $2(k-r-t+1)-3 \geq$ 3 and $\underset{k-r-t+1}{\frac{1}{2}}\left(\begin{array}{ll}0 & 1 \\ 1 & 0\end{array}\right)$ is $Z_{2}$-maximal. Hence $\frac{1}{k-r-t+1} \frac{1}{2}\left(\begin{array}{ll}0 & 1 \\ 1 & 0\end{array}\right)$ represents $A_{r}$ $\perp\left(u_{1}\right),\left(u_{1} \in Z_{2}^{\times}\right) . \quad 2^{-1} \operatorname{det}\left(2 A_{r} \perp\left(2 u_{1}\right)\right) \in Z_{2}^{\times}$implies that this representation is primitive.

(ii) The case of $t=n-2 r-2$; then $k-r+1-t \geq 5$.

$$
\frac{1}{2}(u, 1)\left(\begin{array}{ll}
0 & 1 \\
1 & 0
\end{array}\right)\left(\begin{array}{l}
u \\
1
\end{array}\right)=u, \quad\left(\begin{array}{ll}
0 & 1 \\
1 & 0
\end{array}\right) \perp\left(\begin{array}{ll}
0 & 1 \\
1 & 0
\end{array}\right) \cong\left(\begin{array}{ll}
2 & 1 \\
1 & 2
\end{array}\right) \perp\left(\begin{array}{ll}
2 & 1 \\
1 & 2
\end{array}\right)
$$

yield that $\frac{1}{4} \frac{1}{2}\left(\begin{array}{ll}0 & 1 \\ 1 & 0\end{array}\right)$ represents $A_{r} \perp\left(u_{1}\right) \perp\left(u_{2}\right)$ primitively. Thus we have proved $A_{2}^{\prime}(T) \neq 0$ for $T \in \Lambda_{n}^{*}$.

Q.E.D.

If $S_{p}(T) \geq\left(1-p^{-2}\right)^{4 n}$ for odd prime $p$, then

$$
S_{T}=\prod S_{p}(T) \geq S_{2}(T) \prod_{p \neq 2}\left(1-p^{-2}\right)^{4 n}>\varepsilon^{\prime}>0
$$

holds, and it completes the proof. From now we show

$$
S_{p}(T) \geq\left(1-p^{-2}\right)^{4 n} \quad \text { for odd prime } p, T \in \Lambda_{n}^{*} \text {. }
$$

We fix an odd prime $p$. Let $L$ be a hyperbolic space of $\operatorname{dim} 2 k$ over $F_{p}=Z /(p)$; then $A_{p}^{\prime}(T)$ is equal to the number of isometries from the quadratic space over $F_{p}$ corresponding to $T$ to $L$ where isometries are supposed to be injective. For quadratic spaces $M, N$ over $F_{p}$ we denote by $A(M, N)$ the number of isometries from $M$ to $N$. Our aim is to prove that

$$
A(T, L) \geq p^{2 k n-n(n+1) / 2}\left(1-p^{-2}\right)^{4 n} \quad \text { for any quadratic space }
$$

$T$ of dimension $n$.

Let $T$ be a quadratic space of $\operatorname{dim} n$ over $F_{p}$ and $T=T_{0} \perp R$ where $R$ is the radical of $T$. Define a quadratic space $L_{1}$ by $L \cong T_{0} \perp L_{1}$; then $A(T, L)=A\left(T_{0}, L\right) A\left(R, L_{1}\right)$ and 


$$
A\left(T_{0}, L\right)=p^{-t(t+1) / 2+2 k t} \begin{cases}\left(1 \pm p^{-k}\right)\left(1 \pm p^{-k+t / 2}\right) \prod_{s=1}^{t / 2-1}\left(1-p^{2 s-2 k}\right) & \text { if } t \equiv 0 \bmod 2, \\ \left(1 \pm p^{-k}\right) \prod_{s=1}^{(t-1) / 2}\left(1-p^{2 s-2 k}\right) & \text { if } t \equiv 1 \bmod 2,\end{cases}
$$

where $t=\operatorname{dim} T_{0}$ ([8]).

From $k \geq n+2$ and $n \geq t$ follows

$$
A\left(T_{0}, L\right) \geq p^{-t(t+1) / 2+2 k t}\left(1-p^{-2}\right)^{2 n} .
$$

Since, then, $A(T, L) \geq p^{2 k n-n(n+1) / 2}\left(1-p^{-2}\right)^{4 n}$ follows from $A\left(R, L_{1}\right) \geq$ $p^{-(n-t)(n-t+1) / 2+(2 k-t)(n-t)}\left(1-p^{-2}\right)^{2(n-t)}$, we have only to prove

Lemma 5. Let $M$ be a regular quadratic space over $F_{p}$ of $\operatorname{dim} M=m$ and let $N$ be a totally isotropic quadratic space over $F_{p}$ of $\operatorname{dim} N=s$. Then we have

$$
A(N, M) \geq p^{-s(s+1) / 2+m s}\left(1-p^{-2}\right)^{2 s} \quad \text { if } m \geq 2 s+3 .
$$

Proof. Put $N=F_{p}\left[v_{1}, \cdots, v_{s}\right], M=H \perp M_{1}$ where $H$ is a hyperbolic plane. For quadratic spaces we denote by $Q, B$ associated quadratic forms and bilinear forms $(Q(x)=B(x, x))$. Take a basis $\{e, f\}$ of $H$ such that $Q(e)=Q(f)=0, B(e, f)=1$. Let $\sigma$ be an isometry from $N$ to $M$ such that $\sigma\left(v_{1}\right)=e$, and put $\sigma\left(v_{i}\right)=a_{i} e+b_{i} f+u_{i}\left(a_{i}, b_{i} \in F_{p}, u_{i} \in M_{1}\right)$. Then $B\left(v_{1}, v_{i}\right)=B\left(\sigma\left(v_{1}\right), \sigma\left(v_{i}\right)\right)=b_{i}=0$. Since $\sigma$ is injective, $u_{2}, \cdots, u_{s}$ are linearly independent and $B\left(u_{i}, u_{j}\right)=0$ for $i, j$. If, conversely, $w_{2}, \cdots, w_{s}$ $\in M_{1}$ are linearly independent and $B\left(w_{i}, w_{j}\right)=0$ for $i, j$, then $\mu\left(v_{1}\right)=e$, $\mu\left(v_{i}\right)=a_{i} e+w_{i}\left(a_{i} \in \boldsymbol{F}_{p}, i \geq 2\right)$ define an isometry from $N$ to $M$. Thus we have

$$
A\left(N_{s}, M\right)=p^{s-1} a(M) A\left(N_{s-1}, M_{1}\right),
$$

where $N_{i}$ denotes a totally isotropic quadratic space of $\operatorname{dim} i$ and $a(M)$ is the number of (non-zero) isotropic vectors of $M$.

Put $M=\frac{\perp}{s-1} H \perp M_{0}$; then we have

$$
\begin{aligned}
A\left(N_{s}, M\right) & =p^{s-1} a\left(\frac{\perp}{s-1} H \perp M_{0}\right) A\left(N_{s-1}, \frac{\perp}{s-2} H \perp M_{0}\right) \\
& =\cdots \\
& =p^{s(s-1) / 2} \prod_{i=0}^{s-1} a\left(\frac{1}{i} H \perp M_{0}\right) .
\end{aligned}
$$


If $\operatorname{dim} M_{0} \equiv 1 \bmod 2$, then we have

$$
\begin{aligned}
a\left(\frac{1}{i} H \perp M_{0}\right) & =p^{2 i+m-2(s-1)-1}-1 \\
& \geq p^{2 i+m-2(s-1)-1}\left(1-p^{-2}\right) \\
& \geq p^{2 i+m-2(s-1)-1}\left(1-p^{-2}\right)^{2},
\end{aligned}
$$

since $2 i+m-2(s-1)-1 \geq 2$.

If $\operatorname{dim} M_{0} \equiv 0 \bmod 2$, then we have

$$
a\left(\frac{\perp}{i} H \perp M_{0}\right)= \begin{cases}p^{2 r-1}+p^{r}-p^{r-1}-1 & \text { if } M_{0} \text { is hyperbolic } \\ p^{2 r-1}-p^{r}+p^{r-1}-1 & \text { otherwise }\end{cases}
$$

where

$$
2 r=2 i+m-2(s-1)=\operatorname{dim} \frac{1}{i} H \perp M_{0} .
$$

Hence

$$
a\left(\frac{1}{i} H \perp M_{0}\right)=\left(p^{r} \mp 1\right)\left(p^{r-1} \pm 1\right) \geq p^{2 r-1}\left(1-p^{-2}\right)^{2}
$$

holds.

Thus we have

$$
\begin{aligned}
A\left(N_{s}, M\right) & \geq p^{s(s-1) / 2} \prod_{i=0}^{s-1} p^{2 i+m-2(s-1)-1}\left(1-p^{-2}\right)^{2} \\
& =p^{m s-s(s+1) / 2}\left(1-p^{-2}\right)^{2 s}
\end{aligned}
$$

CoRollaRY. If $n, k$ are natural numbers such that $k \geq 2 n+2, k \equiv 0$ $\bmod 2$ and $f(Z)=\sum a(T) e(\sigma(T Z))\left(Z \in H_{n}\right)$ is a modular form of degree $n$ and weight $k$, then we have

$$
a(T)=O\left(|T|^{k-(n+1) / 2}\right) \quad \text { for } T>0 .
$$

Proof. It is known that there exist cusp forms $f_{r}$ of degree $r$ and weight $k$ such that $f(Z)=\sum_{r=1}^{n-1} E_{n, r}^{k}\left(Z, f_{r}\right)+a E_{n}^{k}(Z)+f_{n}(Z),(a \in C)$ ([3]). Since $a\left({ }^{t} U T U\right)=a(T)$ for $U \in G L(n, Z)$, we may assume that $T$ is in some fixed Siegel domain. Theorem in $\S 2$ and our theorem imply the corollary.

Q.E.D.

$\S 4$.

Let $A$ be an even integral unimodular positive definite symmetric matrix of rank $m$; then $m \equiv 0 \bmod 8$. Put 


$$
\theta_{n}(Z, A)=\sum_{C \in M_{m, n}(Z)} e\left(\frac{1}{2} \sigma\left({ }^{t} C A C Z\right)\right), \quad Z \in H_{n}
$$

Then $\theta_{n}(Z, A)$ is a modular form of degree $n$ and weight $m / 2$. Then there exist cusp forms $f_{r}$ of degree $r$ and weight $m / 2$ such that

$$
\theta_{n}(Z, A)=E_{n}^{m / 2}(Z)+\sum_{r=1}^{n-1} E_{n, r}^{m / 2}\left(Z, f_{r}\right)+f_{n}(Z) \quad \text { for } n \leq m / 4-1
$$

If

$$
E_{n}^{m / 2}(Z)=\sum_{\substack{T \geq 0 \\ T \in A_{n}^{*}}} a_{m / 2, n}(T) e(\sigma(T Z))
$$

then

$$
a_{m / 2, n}(T)=2^{n(m-n+1) / 2} \prod_{t=0}^{n-1} \frac{\pi^{(m-t) / 2}}{\Gamma((m-t) / 2)} \cdot|T|^{(m-n-1) / 2} \prod_{p} S_{p}(T) \quad \text { for } T>0,
$$

where $S_{p}(T)=\sum_{R} e(-\sigma(T R)) \nu(R)^{-m / 2}$ where $R$ runs over all $n \times n$ rational symmetric matrices modulo 1 such that the product $\nu(R)$ of denominators of elementary divisors of $R$ is a power of $p$.

Put

$$
\theta_{n}(Z, A)=\sum_{\substack{T \geq 0 \\ T \in A_{n}^{*}}} N_{n}(T, A) e(\sigma(T Z))
$$

and

$$
E_{n, r}^{m / 2}\left(Z, f_{r}\right)=\sum_{\substack{T \geq 0 \\ T \in A_{n}^{*}}} a\left(T, f_{r}\right) e(\sigma(T Z))
$$

Then we have, summarizing,

Theorem. If $n \leq m / 4-1, T \in \Lambda_{n}^{*}, T>0$, then $N_{n}(T, A)=a_{m / 2, n}(T)+$ $\sum_{r=1}^{n-1} a\left(T, f_{r}\right)+O\left(|T|^{m / 4}\right)$. If, moreover, $T$ runs in any fixed Siegel domain, then

$$
a_{m / 2, n}(T) \sim|T|^{(m-n-1) / 2}, \quad a\left(T, f_{r}\right)=O\left(\left|T_{r}\right|^{-(m / 2-r-1) / 2}|T|^{(m-n-1) / 2}\right),
$$

where $T_{r}$ stands for the upper left $r \times r$ submatrix of $T$.

For $n \times n$ positive definite matrix $S$ we denote by $m(S)$ the minimal value of ${ }^{t} x S x\left(x \in Z^{n}-\{0\}\right)$. It is well known that there is a constant $\mu_{n}$ such that $m(S) \leq \mu_{n} \sqrt[n]{|S|}$ for any $n \times n$ positive definite matrix $S$.

Corollary. If $n \leq m / 4-1$, then 


$$
N_{n}(T, A)=a_{m / 2, n}(T)+O\left(m(T)^{1-m / 4}|T|^{(m-n-1) / 2}\right) \quad \text { for } T>0, T \in \Lambda_{n}^{*} .
$$

Especially $N_{n}(T, A)>0$ if $m(T)$ is sufficiently large.

Proof.

$$
\begin{aligned}
\left|T_{r}\right|^{-(m / 2-r-1) / 2} & =O\left(m\left(T_{r}\right)^{-r(m / 2-r-1) / 2}\right) \\
& =O\left(m(T)^{-r(m / 2-r-1) / 2}\right) .
\end{aligned}
$$

On the other hand

$$
\begin{aligned}
|T|^{m / 4}\left(m(T)^{1-m / 4}|T|^{(m-n-1) / 2}\right)^{-1} & =m(T)^{m / 4-1}|T|^{(2 n+2-m) / 4} \\
& \leq \mu_{n}^{m / 4-1}|T|^{(m / 4-1) / n+(2 n+2-m) / 4} .
\end{aligned}
$$

Since $r(m / 2-r-1) / 2 \geq m / 4-1$ for $1 \leq r \leq n \leq m / 4-1$, we have $a\left(T, f_{r}\right)$ $=O\left(m(T)^{1-m / 4}|T|^{(m-n-1) / 2}\right),|T|^{m / 4}=O\left(m(T)^{1-m / 4}|T|^{(m-n-1) / 2}\right)$, if $|T| \geq 1$.

There are only finitely many equivalence classes of $T \in \Lambda_{n}^{*}$ such that $T>0,|T|<1$. This completes the proof. Q.E.D.

Remark. Let $f(Z)=\sum a(T) e(\sigma(T Z))$ be a modular form of degree $n$, weight $k\left(\in \frac{1}{2} Z\right)$ with level such that the constant term of $f(Z)$ at any cusps vanishes. Results in [2] and here seem to suggest that $a(T)=$ $=O\left(m(T)^{1-k / 2}|T|^{k-(n+1) / 2}\right)$ for $T>0$ if, at least, $2 k \geq 2 n+3$.

\section{REFERENCES}

[1] W. L. Baily, Jr., Introductory lectures on automorphic forms, Iwanami Shoten, Publishers and Princeton University Press (1973).

[2] J. S. Hsia, Y. Kitaoka, and M. Kneser, Representations of positive definite quadratic forms, J. reine angew. Math. 301 (1978), 132-141.

[ 3 ] H. Klingen, Zum Darstellungssatz für Siegelsche Modulformen, Math. Zeitschr. 102 (1967), 30-43.

[ 4 ] M. Kneser, Quadratische Formen, Vorlesungs-Ausarbeitung, Göttingen (1973/4).

[5] H. Maaß, Siegel's modular forms and Dirichlet series, Lecture Notes in Math. 216, Springer-Verlag (1971).

[6] O. T. O'Meara, Introduction to quadratic forms, Springer-Verlag (1963).

[ 7 ] S. Raghavan, Modular forms of degree $n$ and representation by quadratic forms, Ann. of Math. 70 (1959), 446-477.

[ 8 ] C. L. Siegel, Über die analytische Theorie der quadratischen Formen, Ann. of Math. 36 (1935), 527-606.

[ 9 ] C. L. Siegel, Einführung in die Theorie der Modulfunktionen n-ten Grades, Math. Ann. 116 (1939), 617-657. 